\title{
Transcriptome profiling of fruit development and maturation in Chinese white pear (Pyrus bretschneideri Rehd)
}

Min Xie ${ }^{1 \dagger}$, Ying Huang ${ }^{1 \dagger}$, Yanping Zhang ${ }^{1}$, Xin Wang ${ }^{1}$, Hua Yang ${ }^{2}$, Oliver $Y u^{2}$, Wenhao Dai ${ }^{3}$ and Congbing Fang ${ }^{1,2^{*}}$

\begin{abstract}
Background: Pear (Pyrus spp) is an important fruit species worldwide; however, its genetics and genomic information is limited. Combining the Solexa/lllumina RNA-seq high-throughput sequencing approach (RNA-seq) with Digital Gene Expression (DGE) analysis would be a powerful tool for transcriptomic study. This paper reports the transcriptome profiling analysis of Chinese white pear (P. bretschneideri) using RNA-seq and DGE to better understand the molecular mechanisms in fruit development and maturation of Chinese white pear.
\end{abstract}

Results: De novo transcriptome assembly and gene expression analysis of Chinese white pear were performed in an unprecedented depth (5.47 gigabase pairs) using high-throughput Illumina RNA-seq combined with a tag-based Digital Gene Expression (DGE) system. Approximately, 60.77 million reads were sequenced, trimmed, and assembled into 90,227 unigenes. These unigenes comprised 17,619 contigs and 72,608 singletons with an average length of $508 \mathrm{bp}$ and had an $\mathrm{N}_{50}$ of $635 \mathrm{bp}$. Sequence similarity analyses against six public databases (Uniprot, NR, and COGs at NCBI, Pfam, InterPro, and KEGG) found that 61,636 unigenes can be annotated with gene descriptions, conserved protein domains, or gene ontology terms. By BLASTing all 61,636 unigenes in KEGG, a total of 31,215 unigenes were annotated into 121 known metabolic or signaling pathways in which a few primary, intermediate, and secondary metabolic pathways are directly related to pear fruit quality. DGE libraries were constructed for each of the five fruit developmental stages. Variations in gene expression among all developmental stages of pear fruit were significantly different in a large amount of unigenes.

Conclusion: Extensive transcriptome and DGE profiling data at five fruit developmental stages of Chinese white pear have been obtained from a deep sequencing, which provides comprehensive gene expression information at the transcriptional level. This could facilitate understanding of the molecular mechanisms in fruit development and maturation. Such a database can also be used as a public information platform for research on molecular biology and functional genomics in pear and other related species.

\section{Background}

The genus Pyrus is one of the most important genera in Rosaceae family for fruit production. Pyrus species are widely used for commercial fruit production in 76 countries or regions around the world (http://faostat.fao.org) and their economic importance has been well recognized [1]. There are four major edible species in Pyrus: P. communis

\footnotetext{
*Correspondence: fcb_ah@ahau.edu.cn

${ }^{\dagger}$ Equal contributors

'School of Horticulture, Anhui Agricultural University, Hefei 230036, Anhui, P R China

${ }^{2}$ Key Laboratory of Tea Biochemistry \& Biotechnology, Ministry of Education, Anhui Agricultural University, Hefei 230036, Anhui, P R China

Full list of author information is available at the end of the article
}

L. is mainly cultivated in Europe, North America, South America, Africa, and Australia. The other three species, $P$. bretschneideri Rehd., P. ussuriensis Maxim., and P. pyrifolia (Burm.) Nakai., are grown in East Asian [1,2]. The world production of pear has doubled in the past 16 years. China is the largest pear producer. In 2010, China produced 15.23 million tons (Mt) of pear fruits that accounted of $67.26 \%$ of world pear production (22.64 Mt) (FAOSTAT, 2012).

As of July 2013, 26,696 nucleotide sequences, 4,413 expressed sequence tags (ESTs), 52 genome survey sequences (GSS), and 2,636 proteins from the Pyrus genus have been deposited in GenBank. These sequences mostly derived from cDNA cloning and EST sequencing [3-7]

\section{Biomed Central}


provide useful information for transcriptional analysis, candidate gene discovery, and gene functional analysis; however, a comprehensive description of genes that expressed in pear fruit during the fruit development and maturation period remains unavailable.

Recent years, use of RNA-seq, the next generation sequencing technology, has generated over one billion bp of high-quality DNA sequence per analysis/experiment [8] and has dramatically improved the efficiency of gene discovery and functional analysis $[9,10]$, which largely facilitates the investigation of the functional complexity of transcriptomes [11,12]. Illumina sequencing of transcriptomes for yeast [13,14], Arabidopsis [15], mouse [16,17], and human cells $[18,19]$ has confirmed that next generation sequencing is well-suited for surveying transcriptome profiles in eukaryotes. Recently Illumina RNA-seq system has been used to identify genes related to bud dormancy in pear (P. pyrifolia) [20]. RNA-seq is not limited to detecting transcripts for organisms with existing genomic sequences; it also can be used to sequence nonmodel organisms that lack of genomic information [21-25]. DGE is a tag-based transcriptome sequencing approach in which the expression level of all genes in a sample is measured by counting the number of individual mRNA molecules produced from each gene, which enables the DGE protocol more suitable and affordable for comparative gene expression studies [25-31]. Despite its obvious potential, the next generation sequencing has not yet been applied to pear research.

In this study, 5.47 gigabase pairs of high quality DNA sequence were generated using Illumina technology to survey the poly $(\mathrm{A})^{+}$transcriptome of $P$. bretschneideri. A total of 90,227 unigenes were assembled. All known homologous genes in major metabolic pathways related to fruit development and maturation were identified. Furthermore, five DGE libraries were constructed and gene expression profiles at different fruit developmental stages were analyzed. These annotated transcriptome sequences and gene expression profiles provide useful information for identification of genes involved in quality trait development during fruit development and maturation in pear species.

\section{Methods}

\section{Plant material and RNA extraction}

All samples were collected from a 45-year old field grown Pyrus bretschneideri 'Dangshansuli' tree. Tissue samples of tender shoot, young leaf, expanded leaf, mature leaf, flower, and fruit were collected in 2010. Fruit samples were collected at 25, 55, 85, 115 and 145 DPA (days post anthesis) representing five developmental stages (Figure 1). All samples were immediately frozen in liquid nitrogen and kept at $-80^{\circ} \mathrm{C}$ until use. Total RNA was extracted using modified CTAB method [32,33]. RNA quality was monitored using the Agilent 2100 Bioanalyzer with a minimum integrity number value of 8 .

\section{cDNA library construction, Illumina sequencing and De novo assembly}

To obtain a complete gene expression profile, RNA from each tissue sample was pooled. The poly (A) ${ }^{+}$RNA was isolated from $20 \mu \mathrm{g}$ of the pooled RNA using Dynal oligo(dT) 25 beads (Invitrogen) according to the Illumina manufacturer's instructions. The mRNA was then cleaved into short sequences using divalent cations at $70^{\circ} \mathrm{C}$ for $5 \mathrm{~min}$. The cleaved RNA fragments were used for first strand cDNA synthesis using SuperScript III reverse transcriptase (Invitrogen) and N6 random hexamers (Takara). Second strand cDNA synthesis was performed using RNase $\mathrm{H}$ (Invitrogen) and DNA polymerase (Invitrogen). Subsequently, cDNA fragments were ligated to adapters after an end repair process. These products were purified and enriched using the QIAquick PCR Purification Kit to develop a cDNA library.

The cDNA library was sequenced from both $5^{\prime}$ and $3{ }^{\prime}$ ends on the Illumina sequencing platform (HiSeq ${ }^{\mathrm{TM}}$ 2000). Image deconvolution and Q-value (quality value assigned to each base) calculation were carried out in the Illumina data processing pipeline (version 1.6). Before assembly, adaptor sequences, empty reads, low quality sequences with $\mathrm{N}$ percentage (i.e., the percentage of nucleotides in read which could not be sequenced) over $5 \%$, and those containing more than $20 \%$ bases with $\mathrm{Q}$-value $\leq 10$ were removed. De novo assembly of the cleaned reads was performed using SOAPdenovo software (version 1.03, http://soap.genomics.org.cn). The de Bruijn graph was firstly used to generate contigs [34]. The reads were then mapped back to the contigs, and the paired-end reads and contigs from the same transcript were further joined into scaffolds. The complete scaffolds were subsequently obtained after the pairedend reads were again used for gap fillings [34]. To reduce any sequence redundancy, the scaffolds were further assembled into unigenes using TGICL (copyright (c), http://www.tigr.org/tdb/tgi) [35]. Among them, the scaffolds with more than $70 \%$ similarity after multiple alignments were grouped into clusters, and others that cannot reach the threshold set and fall into any assembly should remain as a list of singletons. The assembled unigenes (larger than $200 \mathrm{bp}$ ) were deposited in the Transcriptome Shotgun Assembly Sequence Database (TSA) at NCBI with the accession number from JR595427 to JR673747.

\section{Functional annotation and classification}

Unigenes that were larger than $150 \mathrm{bp}$ were used for BLAST search and annotation against the NCBI 

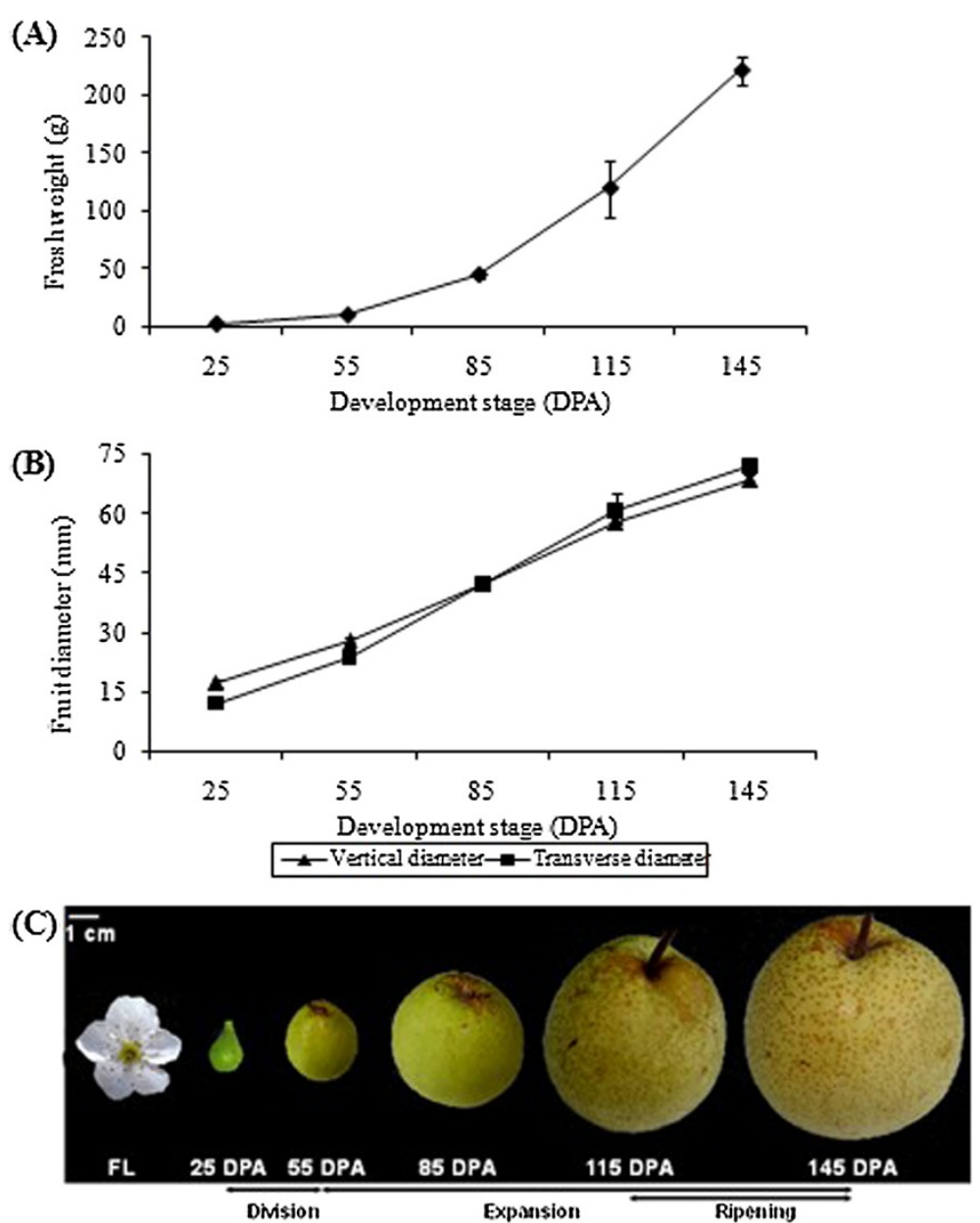

Figure 1 Growth and development of 'Dangshansuli' pear fruit. (A) Changes in fruit fresh weight. Mean of 5-10 fruits. Vertical bars represent standard deviations. (B) Changes in fruit transverse diameter and vertical diameter. Mean of 5-10 fruits. Vertical bars represent standard deviations. (C) Stages of fruit development from flower (FL) to ripening fruit and the corresponding days post anthesis (DPA) separated into 3 phases of development characterized by cell division, cell expansion, and fruit ripening.

nonredundant (nr) database with the BLASTX algorithm (http://www.ncbi.nlm.nih.gov/) using an E-value cut-off of $10^{-5}$. Functional annotation by gene ontology terms (GO; http://www.geneontology.org) was carried out based on the best hits from nr annotation using Blast2go software (http://www.blast2go.de/) [36], and the resultant GO id were further used for GO classification by WEGO [37] (http://wego.genomics.org.cn/cgi-bin/wego/ index.pl). Annotation of COG and KEGG pathways was performed by sequence comparisons using BLASTX algorithm against Cluster of Orthologous Groups database and Kyoto Encyclopedia of Genes and Genomes database with an E-value threshold of $10^{-5}$. Annotations of all assembled sequences were deposited in the Transcriptome Shotgun Assembly Sequence Database (TSA) at NCBI and can be searched using the Gene-ID listed in Additional file 1.

\section{Digital gene expression (DGE) library construction and sequencing}

Total RNA was extracted from fruit samples collected at five fruit developmental stages. The tag library was prepared using the Illumina gene expression sample prep kit. Briefly, the poly $(\mathrm{A})^{+}$RNA was purified from $6 \mu \mathrm{g}$ of RNA using oligo(dT) magnetic beads. Double-stranded cDNA was directly synthesized on the beads and subsequently digested with NlaIII. All cDNA fragments with 3 ' ends were purified with magnetic beads before their $5^{\prime}$ ends were ligated to Illumina adapter 1 . A 21 bp tag with the adaptor 1 was produced after digested with MmeI (an enzyme that recognizes the junction of the Illumina adaptor 1 and the CATG site). The Illumina adapter 2 was then ligated to the 3 ' ends of the tag. The library was amplified by PCR for 15 cycles and $90 \mathrm{bp}$ strips were purified by PAGE gel electrophoresis. These 
strips were then digested and single-chain molecules were attached to the Illumina sequencing chip for sequencing. All raw tag data are available in Gene Expression Omnibus (GEO) at NCBI with the accession number: GSE33388.

\section{Analysis and mapping of DGE tags}

To map DGE tags, sequencing-received raw image data were filtered to remove low quality tags (tags with unknown sequences ' $\mathrm{N}$ '), empty tags (sequence with only the adaptors but no tags), and tags with only one copy number (probable sequencing error). For annotation, cleaned tags with CATG and the 21 bp tag sequence were mapped to our transcriptome reference database with no more than 1 nucleotide mismatch. All tags that mapped to reference sequences of multiple genes were filtered out and the remaining tags were designated as unambiguous tags for gene expression analysis. The number of unambiguous tags of each gene was calculated and then normalized to TPM (number of transcripts per million clean tags). The differentially expressed tags were used for mapping and annotation. A complete list of all differentially expressed genes is shown in Additional file 2, Additional file 3, Additional file 4 and Additional file 5.

\section{Evaluation of DGE libraries}

To compare the gene expression in different fruit developmental stages, the frequency of each tag in different DGE libraries was statistically analyzed using the method of Audic et al. [38]. The false discovery rate (FDR) was used to determine the threshold $P$-value in multiple tests. The threshold determining the significance of differentially expressed genes was set at FDR $\leq 0.001$ and $\log 2$ foldchange $\geq 2$.

\section{Pathway enrichment analysis}

Pathway enrichment analysis helps to identify significantly enriched metabolic pathways or signal transduction pathways in differentially expressed genes (DEGs) compared to the whole genome background. All DEGs mapped in the KEGG pathways with P-value $\leq 0.05$ were considered statistically significantly enriched. The calculating formula is:

$$
\mathbf{P}=1-\sum_{i=0}^{m-1} \frac{\left(\begin{array}{c}
M \\
i
\end{array}\right)\left(\begin{array}{c}
N-M \\
n-i
\end{array}\right)}{\left(\begin{array}{c}
N \\
n
\end{array}\right)}
$$

Here $N$ is the number of all genes that with KEGG annotation, $n$ is the number of DEGs in $N, M$ is the number of all genes that were annotated to specific pathways, and $m$ is number of DEGs in $M$.

\section{Results}

\section{Illumina sequencing and de novo assembly}

To obtain a global and comprehensive overview of the pear transcriptome, RNA was extracted from six different tissues including tender shoots, young leaves, expanded leaves, mature leaves, flowers, and fruits were equally mixed. A total of 60.8 millions of $90 \mathrm{bp}$ raw reads were obtained from the Illumina paired-end sequencing with 5.47 gigabase pairs (Gbp) of raw data. After a stringent quality filtering process, $4.95 \mathrm{Gbp}$ of clean data (90.5\% of raw data) was remained. The Q20 percentage (an error probability lower than $1 \%$ ) of the final sequence generated in this study (97.5\%) was greater than those of tea (88\%) [39], mouse (95\%) [40], and Chinese bayberry (93\%) [41], indicating that sequencing throughput and quality was acceptable for further analysis. De novo assembly of all clean reads by SOAPdenovo program produced 132,987 contigs (Table 1 ). The mean contig size was 338 bp and the $\mathrm{N}_{50}$ was 474 bp (i.e. $50 \%$ of the assembled bases were incorporated into contigs 474 bp or longer). Of all 132,987 contigs, 25,936 (19.5\% of total) were larger than 500 bp (Figure 2A). After paired-end joining and gap-filling, the contigs were subsequently assembled into scaffolds. To reduce sequence redundancy, the assembled scaffolds were further clustered into 90,227 unigenes (larger than $150 \mathrm{bp}$ ) using TGICL software [35], including 17,619 clusters (mean size: $732 \mathrm{bp}, \mathrm{N}_{50}$ : $902 \mathrm{bp}$ ) and 72,608 singletons (mean size: $452 \mathrm{bp}, \mathrm{N}_{50}$ : $550 \mathrm{bp}$ ), as shown in Table 1. This unigene set had an average length of $508 \mathrm{bp}$ and an $\mathrm{N}_{50}$ of 635 bp (Table 1). Of all 90,227 unigenes, 25,415 (28.2\%) were $\geq 500$ bp and $8,452(9.4 \%)$ were $\geq 1,000 \mathrm{bp}$. The size distribution of these unigenes is shown in Figure 2B. To evaluate the quality of the dataset, the sequencing bias was analyzed by detecting random distribution of reads in assembled unigenes (Additional file 6). The $3^{\prime}$ and 5' ends of all assembled unigenes contained relatively fewer reads and other positions showed a greater distribution, indicating that the assembled 90,227 unigenes show a great reliability and likely cover most of the transcriptome sequences.

\section{Annotation of predicted proteins}

Distinct gene sequences were first searched using BLASTx against the non-redundant (nr) NCBI nucleotide database with a cut-off E-value of $10^{-5}$. A total of 61,624 (68.3\% of all distinct sequences) unigenes had a BLAST result above cut-off (Table 2, Additional file 1).

Figure 3 indicates that the longer the assembled sequences, the greater the percentage of sequences with significant matches in the $\mathrm{nr}$ database. As shown in Figure $3 \mathrm{~A}$, only $54.9 \%$ of the unigenes shorter than 500 bp returned significant BLAST scores in the nr database. In contrast, the percentage of unigenes with significant 
Table 1 Summary of sequence assembly of $P$. bretschneideri mRNA pooled from ten different tissues samples

\begin{tabular}{|c|c|c|c|c|}
\hline & Sequences $(n)$ & Base pairs (bp) & Mean length (bp) & N50 (bp) \\
\hline Raw sequencing reads & $60,771,588$ & $5,469,442,920$ & 90 & - \\
\hline Clean reads & $55,015,340$ & $4,951,380,600$ & 90 & - \\
\hline Contigs ( $\geq 100 \mathrm{bp}$ ) & 132,987 & $44,990,137$ & 338 & 474 \\
\hline Clusters ( $\geq 150 \mathrm{bp}$ ) & 17,619 & $13,097,373$ & 732 & 902 \\
\hline Singletons ( $\geq 150 \mathrm{bp}$ ) & 72,608 & $32,694,777$ & 452 & 550 \\
\hline Total unigenes ( $\geq 150 \mathrm{bp}$ ) & 90,227 & $45,796,661$ & 508 & 635 \\
\hline
\end{tabular}

BLAST scores increased sharply in which $88.3 \%$ for query sequences between 500 and 1,000 bp, 97.0\% for query sequences between 1,000 and $1,500 \mathrm{bp}, 99.2 \%$ for query sequences between 1,500 and $2,000 \mathrm{bp}$, and $100 \%$ for query sequences $\geq 2,000 \mathrm{bp}$. The E-value distribution of the top hits in the nr database showed that $34.0 \%$ of the mapped sequences have a great similarity (smaller than $1.0 \mathrm{E}-50$ ) with $66.0 \%$ of the sequences ranged from $1.0 \mathrm{E}-5$ to $1.0 \mathrm{E}-50$ (Figure $3 \mathrm{~B}$ ). Furthermore, $25.4 \%$ of the query sequences have a similarity higher than $80 \%$, while $74.6 \%$ of the hits have a similarity ranging from $20 \%$ to $80 \%$ (Figure 3C). Among species, 36.2\% of pear distinct sequences have top matches (first hit) with sequences from Arabidopsis thaliana, while only 7.94\%, 7.92\%, $7.86 \%$, and $5.73 \%$ of pear distinct sequences matched to those of Oryza sativa, Populus trichocarpa, Arabidopsis lyrata, and Vitis vinifera, respectively (Figure 3D).

\section{Conserved domain annotation}

Conserved protein domains were identified in the $P$. bretschneideri unigenes against the InterPro, Pfam, and COGs databases. Searches against the InterPro database [42] revealed 32,098 top hits that were categorized into 4,264 domains/families (Table 2). Most domains contain 1-3 sequences, with a small proportion appearing more frequently. InterPro domains/families were ranked according to the number of unigenes included in each InterPro domain group. The 30 most abundant InterPro domains/families are provided in Table 3. Protein kinase and its subcategories Serine/threonine-protein kinase, Tyrosine-protein kinase, and Protein kinase-ATP binding site known to regulate the majority of cellular pathways were ranked in the top conserved domains. Cytochrome P450 and Myb-DNA-binding families that might contribute to extensive modifications of various secondary compounds and the "WD40-repeat" domain that is associated with the regulation of signal transduction, transcription, and proliferation [43] were also highly represented. By searching the Pfam database [44], 30,985 of the assembled unigenes matched entries that are corresponding to 3,406 different domains/families (Table 2).

\section{Gene ontology (GO), clusters of orthologous groups} (COG) and Kyoto Encyclopedia of genes and genomes (KEGG) ontology (KO) classification

Gene ontology (GO) assignments were used to classify functions of the predicted pear genes. Based on the sequence similarity, 28,114 sequences were categorized into 44 functional groups (Figure 4, Additional file 7) in three main categories (biological process, cellular component, and molecular function). Cellular process, metabolic process, cell, cell part, organelle, binding, and catalytic activity are most dominant terms presented in the three categories. Very few genes were clustered into "Biological adhesion", "Cell killing", "Locomoting",
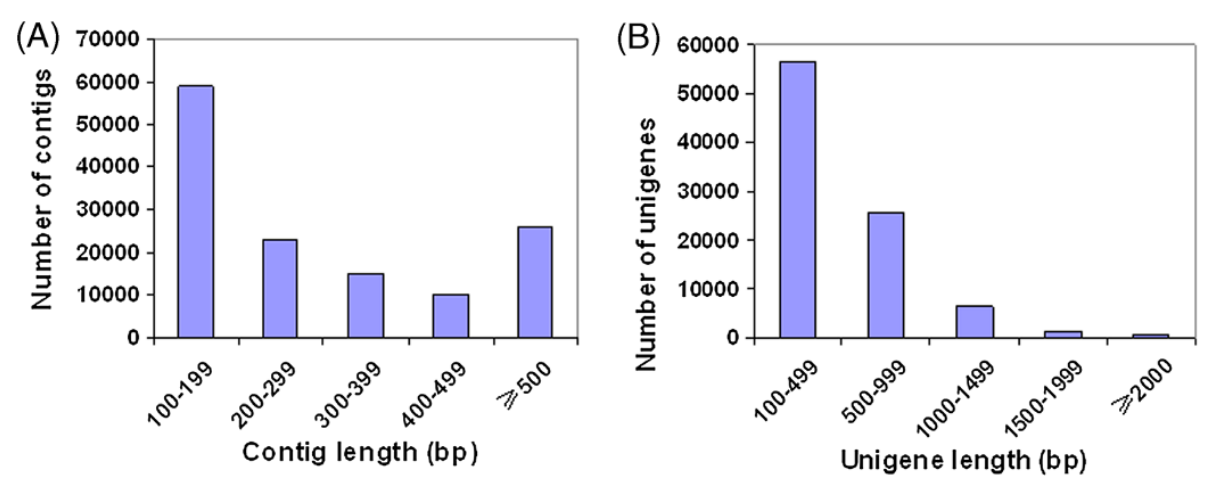

Figure 2 Overview of the P. bretschneideri transcriptome sequencing and assembly. (A) Size distribution of the contigs obtained from de novo assembly of high quality, clean reads. (B) Size distribution of the unigenes produced from further assembly of contigs by contig joining, gap filling, and scaffold clustering. 
Table 2 Summary of annotations of the $P$. bretschneideri unigenes

\begin{tabular}{|c|c|c|c|}
\hline & Sequences $(n)$ & Annotations (n) & Functional classification \\
\hline All assembled unigenes & 90,227 & - & - \\
\hline Gene annotations against NR plant proteins & 61,624 & 29,942 & - \\
\hline Gene annotations against NR Arabidopsis proteins & 27,152 & 36,977 & \\
\hline Unique gene annotations against NR & 61,636 & 61,636 & \\
\hline Gene annotations against UniProt & 40,796 & 40,796 & - \\
\hline Gene annotations against InterPro & 32,098 & 56,450 & 4,264 domains/families \\
\hline Gene annotations against Pfam & 30,985 & 37,194 & 3,406 domains/families \\
\hline Gene annotations against COG & 17,008 & 33,205 & 25 categories \\
\hline Gene annotations against KEGG & 31,215 & 31,215 & 121 pathways \\
\hline GO annotations for NR protein hits & 132,166 & 98,774 & 3 main categories, 44 sub-categories \\
\hline All annotated Unigenes & 62,077 & - & - \\
\hline
\end{tabular}

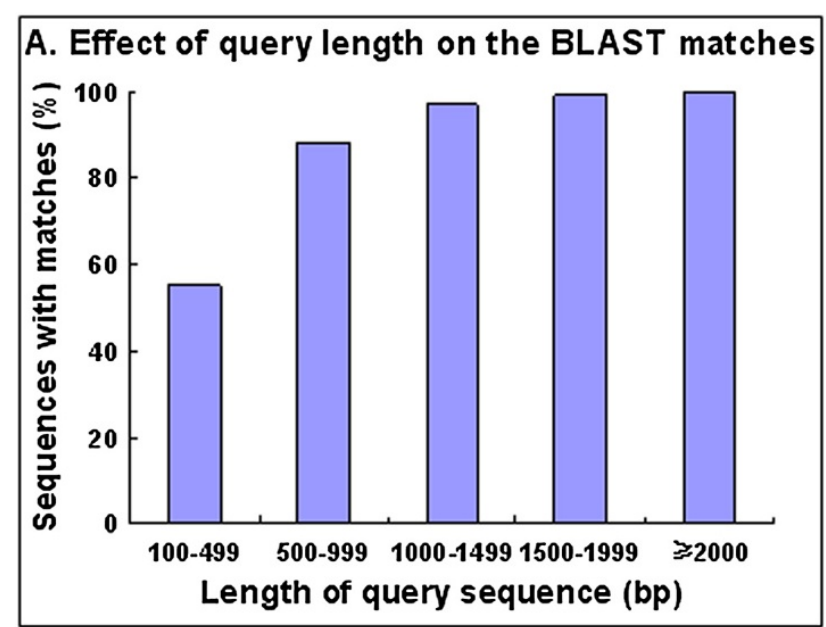

\section{Similarity distribution}

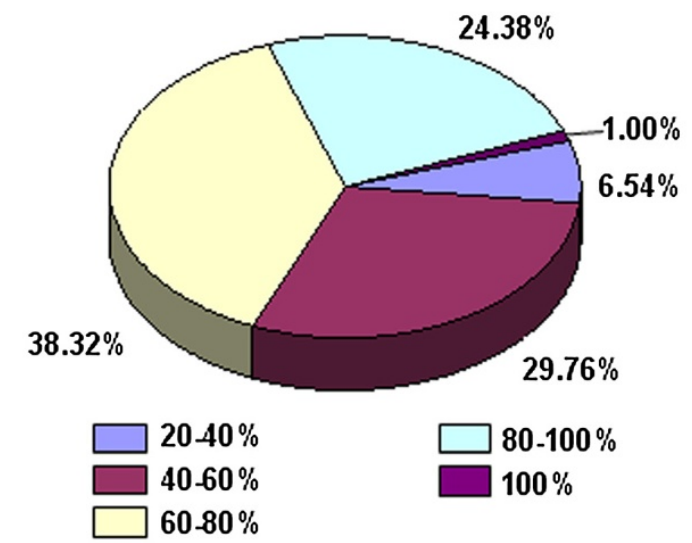

B. E-value distribution
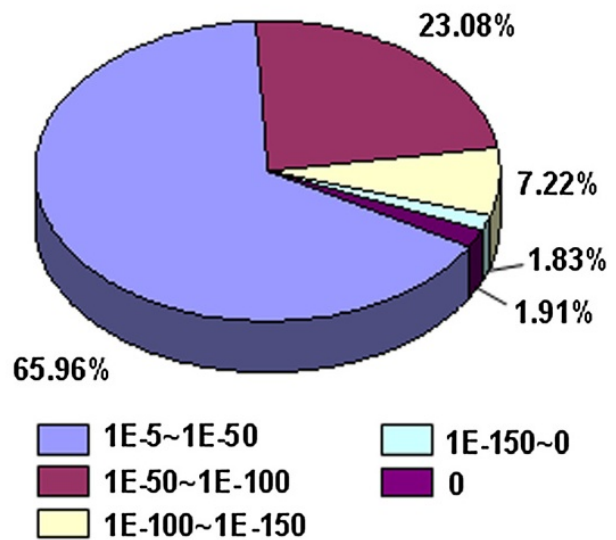

D. Species distribution

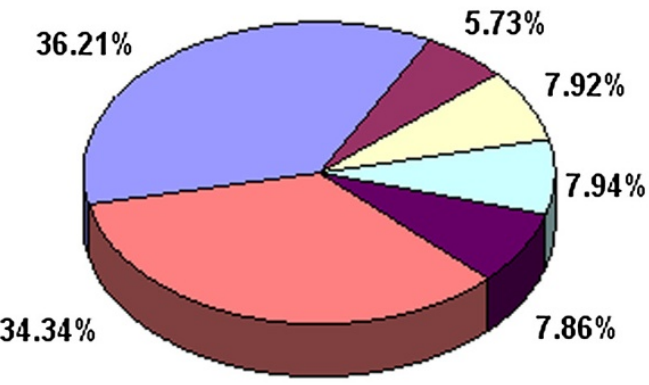

Arabidopsis thaliana

Vitis vinifera

Populus trichocarpa

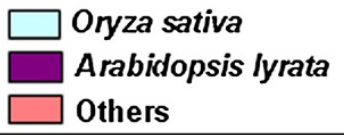

Figure 3 Gene similarity of unigenes against the $\mathrm{nr}$ database. (A) Effect of query sequence length on the percentage of significant matches. The cut-off value was set at 1.0E-5. The proportion of sequences with matches in the $\mathrm{nr}$ database at NCBI is greater among the assembled sequences with a greater length. (B) E-value distribution of the top BLAST hits for each unigene (E-value of 1.0E-5). (C) Similarity distribution of the best BLAST hits for each unigene. (D) Distribution of BLAST results by species is shown as the percentage of the total homologous sequences (with an E-value $\leq 1.0 \mathrm{E}-5$ ). All plant proteins in the NCBI nr database were used for homology search and the best hit of each sequence was used for analysis. 
Table 3 Thirty most frequently occurring InterPro domains/families in the $\boldsymbol{P}$. bretschneideri unigenes

\begin{tabular}{|c|c|c|c|}
\hline Conserved domain/family & Accession ID & Sequences (n) & Rank \\
\hline Protein kinase, catalytic domain & IPR000719 & 1436 & 1 \\
\hline Serine/threonine-protein kinase-like domain & IPR017442 & 958 & 2 \\
\hline Leucine-rich repeat & IPR001611 & 890 & 3 \\
\hline Serine/threonine-protein kinase domain & IPR002290 & 699 & 4 \\
\hline Serine/threonine-protein kinase, active site & IPR008271 & 617 & 5 \\
\hline Pentatricopeptide repeat & IPR002885 & 590 & 6 \\
\hline Tyrosine-protein kinase, catalytic domain & IPR020635 & 589 & 7 \\
\hline Protein kinase, ATP binding site & IPR017441 & 515 & 8 \\
\hline Serine-threonine/tyrosine-protein kinase & IPR001245 & 510 & 9 \\
\hline RNA recognition motif domain & IPR000504 & 351 & 10 \\
\hline WD40 repeat, subgroup & IPR019781 & 348 & 11 \\
\hline Zinc finger, RING-type & IPR001841 & 344 & 12 \\
\hline Cytochrome P450 & IPR001128 & 337 & 13 \\
\hline WD40-repeat-containing domain & IPR017986 & 332 & 14 \\
\hline NB-ARC & IPR002182 & 315 & 15 \\
\hline WD40 repeat 2 & IPR019782 & 309 & 16 \\
\hline WD40 repeat & IPR001680 & 305 & 17 \\
\hline Zinc finger, C3HC4 RING-type & IPR018957 & 250 & 18 \\
\hline Myb, DNA-binding & IPR014778 & 207 & 19 \\
\hline Leucine-rich repeat-containing $\mathrm{N}$-terminal, type 2 & IPR013210 & 194 & 20 \\
\hline HTH transcriptional regulator, Myb-type, DNA-binding & IPR017930 & 187 & 21 \\
\hline SANT domain, DNA binding & IPR001005 & 181 & 22 \\
\hline EF-HAND 2 & IPR018249 & 177 & 23 \\
\hline F-box domain, cyclin-like & IPR001810 & 165 & 24 \\
\hline Cytochrome P450, E-class, group I & IPR002401 & 163 & 25 \\
\hline EF-Hand 1, calcium-binding site & IPR018247 & 162 & 26 \\
\hline Tetratricopeptide repeat-containing & IPR013026 & 161 & 27 \\
\hline WD40 repeat, conserved site & IPR019775 & 156 & 28 \\
\hline Ankyrin repeat-containing domain & IPR020683 & 152 & 29 \\
\hline Helicase, superfamily $1 / 2$, ATP-binding domain & IPR014021 & 148 & 30 \\
\hline
\end{tabular}

"Nitrogen utilization", "Pigmentation", "Rhythmic process", "Extracellular region part", "Virion" or "Antioxidant activity". It is also noticed that a high percentage of genes was clustered in the categories of "Biological regulation", "Developmental process", "Response to stimulus", and "Transporter activity" (Figure 4, Additional file 7).

To further evaluate the completeness of the pear transcriptome library and the effectiveness of the annotation process, a search was conducted to compare the annotated sequences against the COG database. A total of 33,205 annotated sequences were clustered into 25 COG categories (Figure 5) in which the cluster of "General function prediction" represented the largest group (5,021, 15.1\%) followed by "Transcription" (3,230, 9.7\%), "Replication, recombination and repair" (2,660, 8.0\%), and "Posttranslational modification, protein turnover, and chaperones" (2,539, 7.7\%).
Nuclear structure (5, 0.015\%), Extracellular structures (24, $0.072 \%)$, RNA processing and modification (251, 0.8\%), and cell motility $(263,0.8 \%)$ appeared to be the smallest groups (Figure 5, Additional file 8).

A total of 90,227 annotated sequences were mapped to canonical pathways in Kyoto Encyclopedia of Genes and Genomes (KEGG) [45] to identify active pathways in white pear. Of which, 24,169 sequences were assigned to 121 KEGG pathways (Additional file 9). The most represented pathways by the unique sequences were metabolic pathways (5,488 members), biosynthesis of secondary metabolites (2,904 members), plant-pathogen interaction (2,389 members), and spliceosome (1,121 members). These annotations provide a valuable resource for investigating specific processes, functions, and pathways in pear growth and development. 


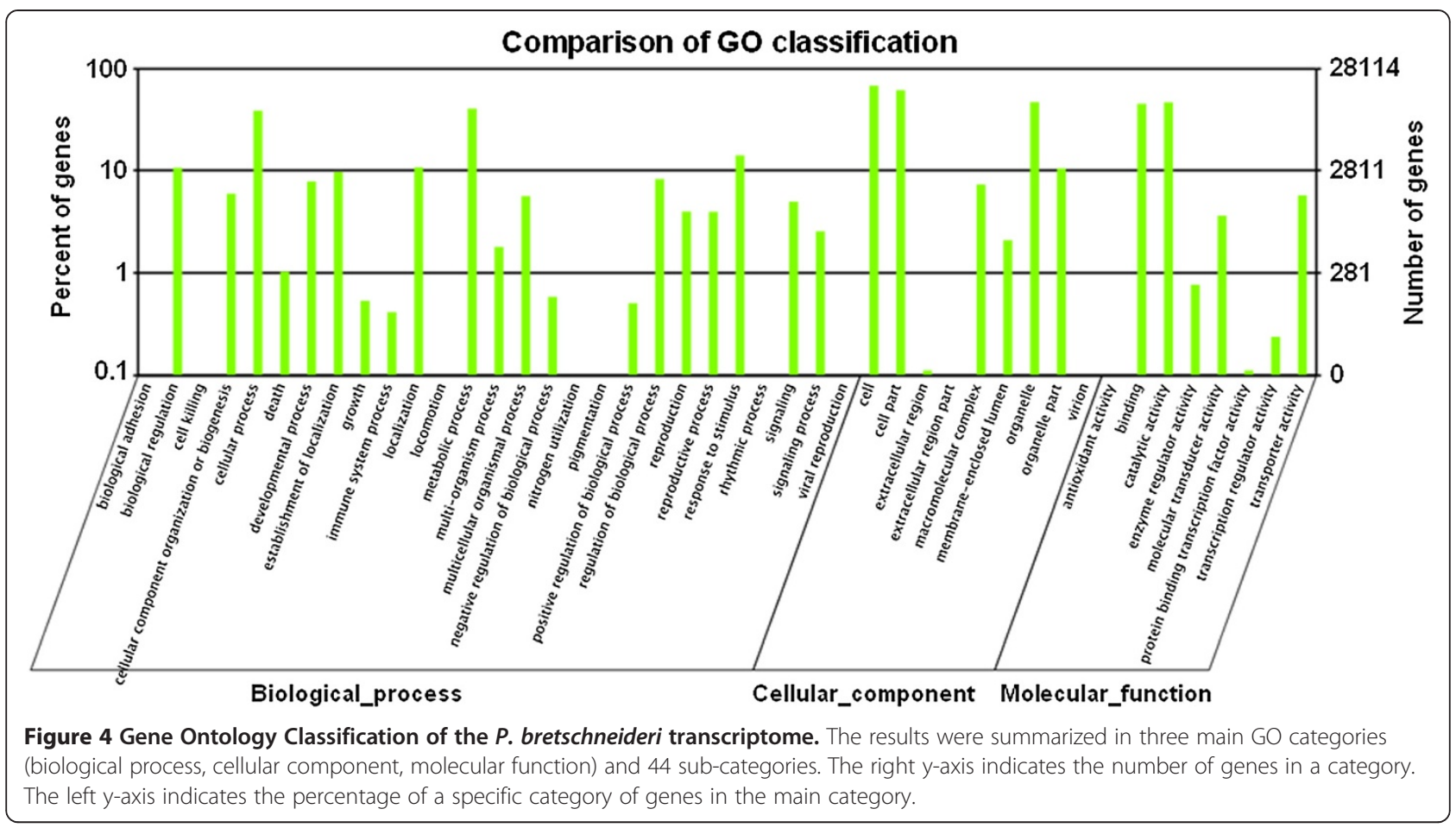

\section{Analysis of metabolic pathway genes using}

\section{$P$. bretschneideri unigenes}

The overall quality of pear fruits is closely related to flavor, grit (stone cell) content, flesh texture, skin russet, and appearance $[1,46]$. During fruit development and maturation, pear fruits undergo a series of physiological and biochemical changes including expansion of size, accumulation of soluble solids, change of pigments, and formation of aromatic volatiles [1,2]. Most of these traits are inherited in a complex polygenic manner and controlled by multiple QTLs, which poses a significant challenge to traditional breeding [2,47]. In order to better understand the genetic and molecular basis of these changes related to quality formation, a few pathways including several primary, intermediate, and secondary metabolic pathways that are related to pear quality development were analyzed. A total of 61,636 annotated unigenes were used to analyze metabolic pathway genes

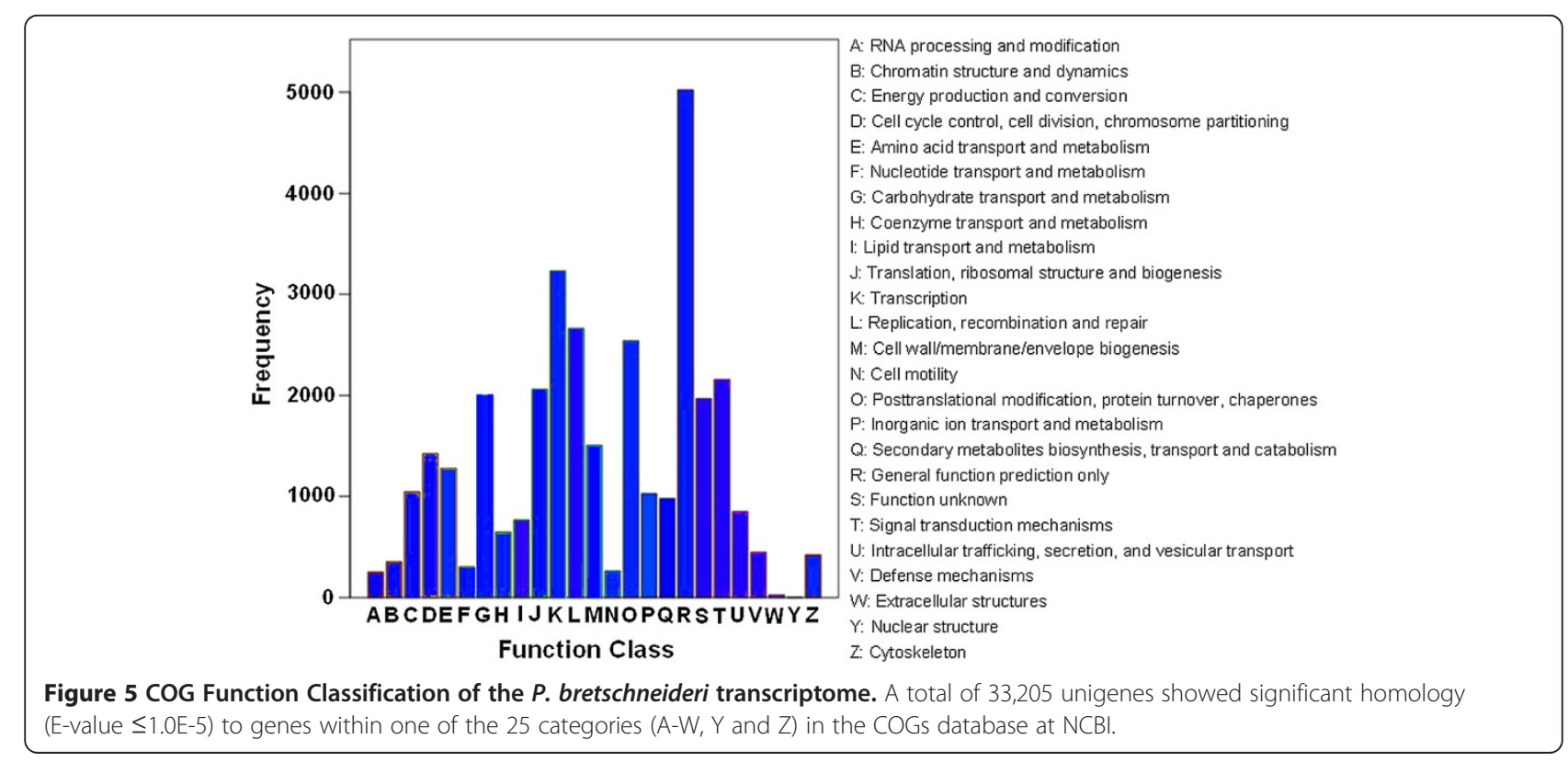


with simple keyword searches. Each search result was confirmed with a BLAST search.

Fruit development is involved in accumulation of starch and soluble sugar in fruits. Investigation of gene expression patterns in carbohydrate metabolisms will facilitate understanding of the fruit quality formation during the course of fruit development. According to KEGG, 2,417 unigenes were identified to be associated with carbohydrate metabolisms. These genes were classified into several metabolic pathways (Figure 6A). In this study, multiple unigenes involved in the main flavonoid biosynthesis pathways were annotated according to KEGG (Figure 6B). Flavonoids including phenylpropanoids, flavones, flavonols, and anthocyanins are important secondary metabolites that are directly involved in pear fruit quality development, such as grit (stone cell) content, skin russet, and appearance $[1,46]$. Starch and sucrose metabolic pathways accounted for $29.2 \%$ of all 2,417 unigenes are another important group of carbohydrate metabolic pathways (Figure 6A). BLAST analysis showed that 18 key enzymes (outlined in Additional file 10) that are defined by 220 unigenes are involved in starch and sucrose metabolism pathways. Invertase (25), sucrose synthase (SS) (24), ADP glucose pyrophosphorylase (24), sucrose phosphate synthase (SPS) (23), and starch synthase (23) were ranked top five greatest numbers of singletons matching the gene description. Other primary metabolic pathways include glycolysis (150 genes), tricarboxylic acid cycle ( 89 genes), and pentose phosphate cycle (66 genes) pathways. The dataset also showed that the annotated sequences in the rest of three primary metabolic pathways have 0 to 25 singletons matching each gene (see Additional file 10). The shikimic acid pathway and the general phenylpropanoid pathway were the intermediate pathways of known topology involved in providing precursors for biosynthesis of flavonoids. A total of 114 unigenes were annotated in 16 enzymes that are involved in shikimic acid or aromatic amino acid pathway. Other 32 unigenes were involved in the general phenylpropanoid pathway and these unigenes might be involved in lignin biosynthesis (Additional file 10). The secondary metabolic pathways were selected, in which the simple six-carbon structure of hexoses were converted into a more complex 6:3:6 basic carbon structure of flavonoids. In the annotated $P$. bretschneideri transcriptome dataset, multiple unigenes encoding almost all known enzymes that are involved in main flavonoid biosynthesis pathways were identified (Additional file 10). All unigenes searched against the transcriptome database of $P$. bretschneideri will facilitate functional genomic studies and are particularly valuable for identifying genes or markers used for molecular breeding of pear species.

\section{Digital gene expression (DGE) library sequencing}

Digital gene expression (DGE) method generates direct gene expression measurements, which avoids the inherent limitation of microarray analysis. Five DGE libraries corresponding to five developmental stages of pear fruits

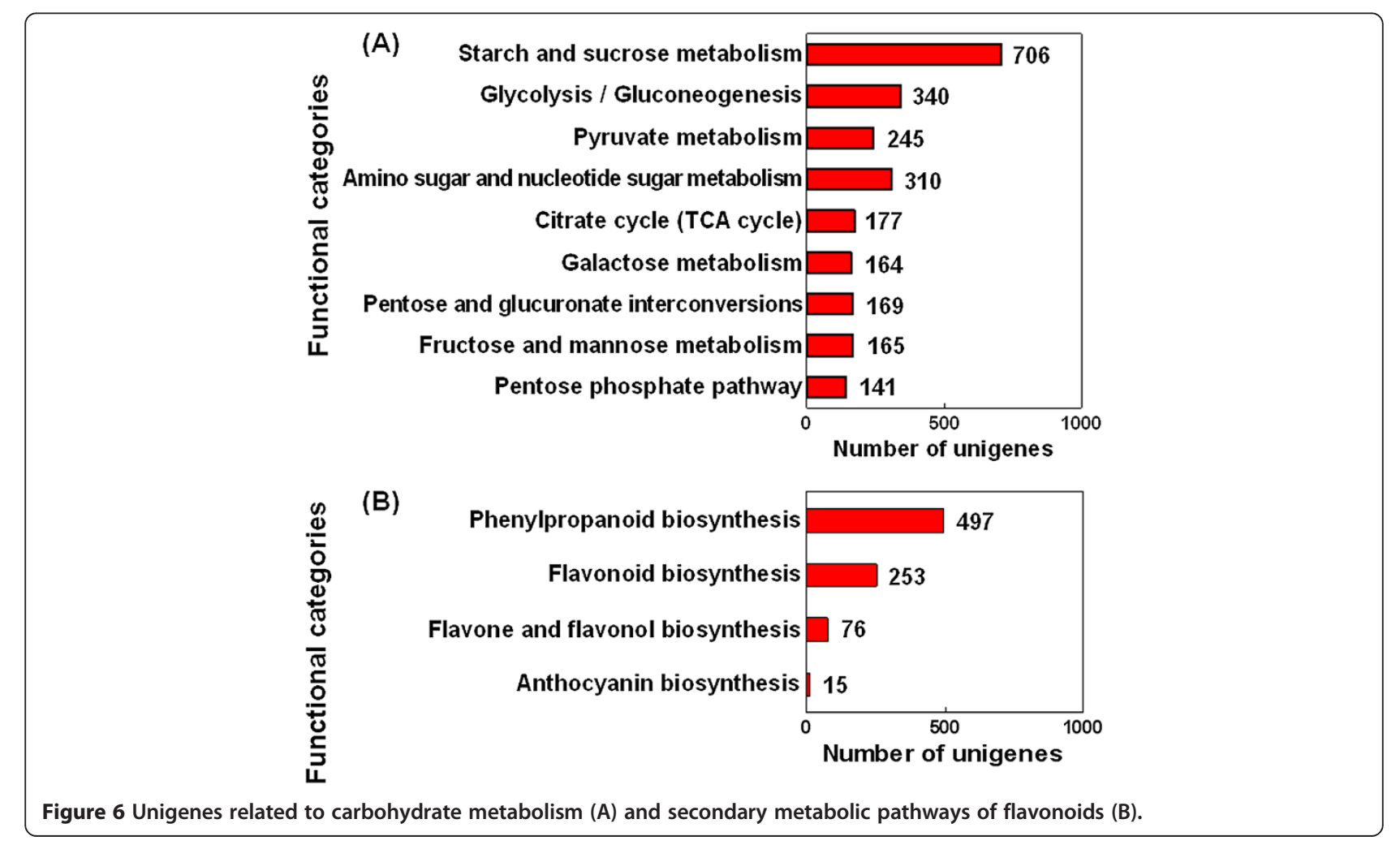


were sequenced with 5.7 to 6.2 million raw tags per library (Table 4). Five stages and their DGE accession numbers deposited in GEO were: fruit stage 1 (GSM825779), fruit stage 2 (GSM825780), fruit stage 3 (GSM825781), fruit stage 4 (GSM825782), and fruit stage 5 (GSM825783). The number of clean tags per library ranged from 5.6 to 6.0 million after filtering out the low quality reads (Figure 7). Of all clean tags, 4.5 to 4.9 million tags were mapped to unigenes (Table 4). The total number of tags with unique nucleotide sequences ranged from 122,997 to 130,176 (Table 4).

During gene expression, heterogeneity and redundancy of mRNA are two significant characteristics with the majority of mRNA being expressed at a low level. To evaluate the normality of the DGE data, distribution of the clean tag expression was evaluated (Figure 8). All five DGE libraries showed a similar pattern in distribution of total tags or distinct tags (2-5 copies, $6-10$ copies, etc., as indicated by different colors). The highly expressed tags (copy number $>100$; Figure 8A) represented greater than $74.9 \%$ of the clean tags in each library; however, the number of high copy number clean tags that appeared in one developmental stage (hence, distinct Figure 8B) did not exceed 7.3\%. In contrast, tags with a low copy number $(<5$; Figure $8 \mathrm{~A})$ represented the majority of distinct clean tags in each library (Figure $8 \mathrm{~B}$ ).

Tag sequences in the five DGE libraries were mapped to the transcriptome reference database generated in the above-mentioned Illumina sequencing. The reference database contains 162,456 distinct sequences with 142,331 unambiguous reference tags. Among the distinct tags $(68,916$ to 77,039$)$ generated from the Illumina sequencing in the five libraries of fruit developmental stages, 32,705 to 36,335 distinct tags were mapped to individual genes in the reference database (Table 4). Tags mapped to a unique sequence are the most critical subset of the DGE libraries as they can explicitly identify a transcript. Up to $31.2 \%(28,175)$ of the sequences in the transcriptome reference tag database could be unequivocally identified by a unique tag (Table 4).

To confirm a proportional increase of the number of detected genes to an increase of total tag number, a saturation analysis was performed. Additional file 11 shows a trend of saturation where the increase of the number of detected genes stopped when the number of reads reached 4 million. The gene expression level was determined by calculating the number of unambiguous tags of each gene and then normalizing the expression level

Table 4 Statistics of $P$. bretschneideri DGE sequencing

\begin{tabular}{|c|c|c|c|c|c|}
\hline Summary & FS1 & FS2 & FS3 & FS4 & FS5 \\
\hline Total number of raw tags & $5,942,273$ & $5,765,350$ & $5,930,561$ & $6,154,708$ & $6,144,427$ \\
\hline Total number of distinct raw tags & 275,061 & 279,671 & 273,457 & 278,272 & 284,436 \\
\hline Total number of clean tags & $5,779,213$ & $5,602,344$ & $5,771,830$ & $5,987,439$ & $5,968,618$ \\
\hline Total number of distinct clean tags & 127,209 & 130,176 & 128,372 & 125,656 & 122,997 \\
\hline Clean tag/Raw tag & $97.26 \%$ & $96.40 \%$ & $97.32 \%$ & $97.28 \%$ & $97.14 \%$ \\
\hline Total number of tags mapping to genes & $4,601,197$ & $4,514,247$ & $4,742,242$ & $4,916,898$ & $4,676,676$ \\
\hline$\%$ of tags mapping to genes & 79.62 & 80.58 & 82.18 & 82.12 & 78.35 \\
\hline Total number of distinct tags mapping to gene & 73,993 & 76,128 & 77,039 & 74,448 & 68,916 \\
\hline$\%$ of distinct tags mapping to gene & 58.17 & 58.48 & 60.01 & 59.25 & 56.03 \\
\hline Number of all tag-mapped genes & 34,781 & 35,530 & 36,335 & 35,415 & 32,705 \\
\hline$\%$ of all tag-mapped genes & 38.55 & 39.38 & 40.27 & 39.25 & 36.25 \\
\hline Number of unambiguous tags mapping to gene & $3,875,820$ & $3,766,999$ & $3,956,567$ & $4,137,841$ & $3,856,928$ \\
\hline$\%$ of unambiguous tags mapping to gene & 67.06 & 67.24 & 68.55 & 69,11 & 64.62 \\
\hline Number of distinct unambiguous tags mapping to gene & 61,872 & 63,617 & 64,242 & 61,873 & 56,894 \\
\hline$\%$ of distinct unambiguous tags mapping to gene & 48.64 & 48.87 & 50.04 & 49.24 & 46.26 \\
\hline Number of unambiguous tag-mapped genes & 26,836 & 27,541 & 28,175 & 27,331 & 24,975 \\
\hline$\%$ of unambiguous tag-mapped genes & 29.74 & 30.52 & 31.23 & 30.29 & 27.68 \\
\hline Number of unknown tags & $1,178,016$ & $1,088,097$ & $1,028,588$ & $1,070,541$ & $1,291,942$ \\
\hline$\%$ of unknown tags & 20.38 & 19.42 & 17.82 & 17.88 & 21.65 \\
\hline Number of distinct unknown tags & 53,216 & 54,048 & 51,333 & 51,208 & 54,081 \\
\hline$\%$ of distinct unknown tags & 41.83 & 41.52 & 39.99 & 40.75 & 43.97 \\
\hline
\end{tabular}

Notes: Statistics of raw tags, clean tags, tags mapped to unigenes, unambiguous tags and unknown tags. Abbreviations: DGE, digital gene expression; FS1, fruit stage 1 ; FS2, fruit stage 2; FS3, fruit stage 3; FS4, fruit stage 4; FS5, fruit stage 5. 


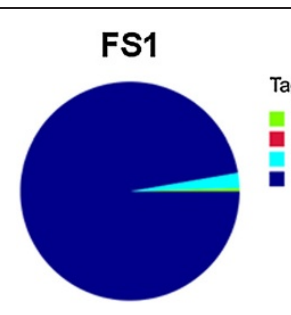

FS3

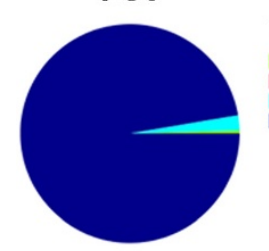

Tag Classification

- Tags Containing N $(25873,0.44 \%)$ Only adaptors $(220,0.00 \%)$ Copy Number < 2 (132638, 2.24\%) Clean tags $(5771830,97.32 \%)$

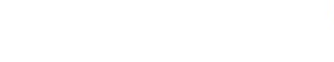

FS5

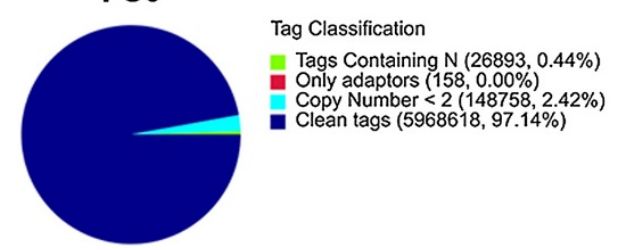

FS2

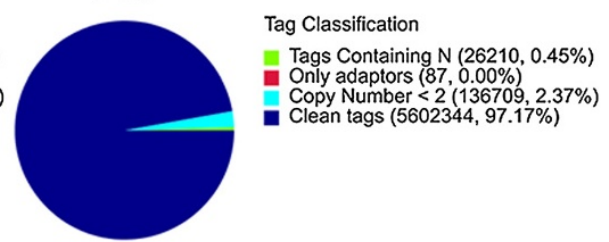

FS4

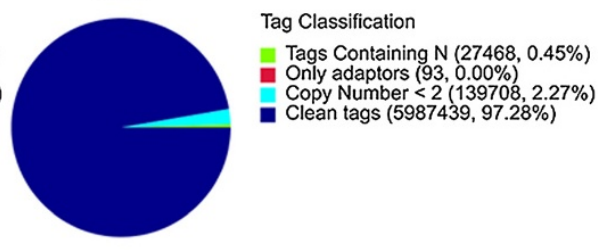

Figure 7 Different components of the raw tags in each pear fruit sample. Percentages of tags containing $\mathrm{N}$ (tags with unknown sequences), adaptors, a tag copy number $<2$, clean tags, and raw tags in each fruit stage library. The numbers in parentheses indicate the percentage of each type of tag among the total raw tags.

to the number of transcripts per million tags (TPM). As summarized in Figure 9, the majority of genes (those toward the right of each graph) resulted in fewer than ten copies and only a small proportion of genes are highly expressed.

\section{Gene expression profile changes between developmental stages}

To profile the gene expression pattern during different developmental stages of pear fruit, the number of clean tags for each gene was calculated and its differentially expressed tags between two samples of two adjacent stages were identified using an algorithm developed by Audic et al. [38].

A total of 2,980 tags were detected to be significantly changed between fruit stage 2 (FS2) and fruit stage 1 (FS1) libraries. Those tags were mapped to 810 genes with 478 genes being up-regulated and 332 genes being downregulated (Figure 10, Additional file 2). Five of the ten most up-regulated genes have defined functions: a dehydrationinduced RD22-like protein, a metallothionein-like protein type 3, a nonspecific lipid-transfer protein precursor, a cytochrome P450, and a Cytochrome P450 82A4. Only one of the ten most down-regulated genes has defined functions: a zinc finger protein. In addition, a total of 14 genes among the 20 differentially expressed genes had unknown functions or no annotations (Additional file 12). According to the GO classification, most of the genes are correlated to cellular components: cytoplasm, plastid, organelle, or intracellular organelle. In the KO classification, 119 gene sets were significantly enriched and most of these genes were correlated to metabolic processes, i.e., metabolic pathways, photosynthesis-antenna proteins, and photosynthesis (Additional file 13).

The comparative analysis between fruit stage 3 (FS3) and FS2 libraries revealed that 2,135 genes showed significant expression changes. Among these genes, 923 and 1,212 genes were up-regulated and down-regulated, respectively, in FS3 compared to FS2 (Figure 10, Additional file 3). Among the ten most up-regulated and ten most downregulated genes, seven annotated genes were up-regulated in FS3: two prunin 2 precursor [Prunus dulcis], one unnamed protein product [Vitis vinifera], one dehydrationresponsive protein RD22 [Prunus persica], one mitochondrial carrier protein [Ricinus communis], one conserved hypothetical protein [Ricinus communis], and one tonoplast intrinsic protein (putative) [Ricinus communis]. Seven functionally defined genes were down-regulated in FS3, including a flavonoid 3-hydroxylase gene [Ricinus communis], a RNA binding protein (putative) [Ricinus communis], an APETALA2 (AP2) domain class transcription factor [Malus x domestica], a CBF/DREB1 transcription factor [Malus baccata], an anthocyanidin synthase gene [Pyrus communis], an Auxin-binding protein ABP19a, and an NADH dehydrogenase subunit 6 [Nicotiana tabacum]. Six genes were also blasted 


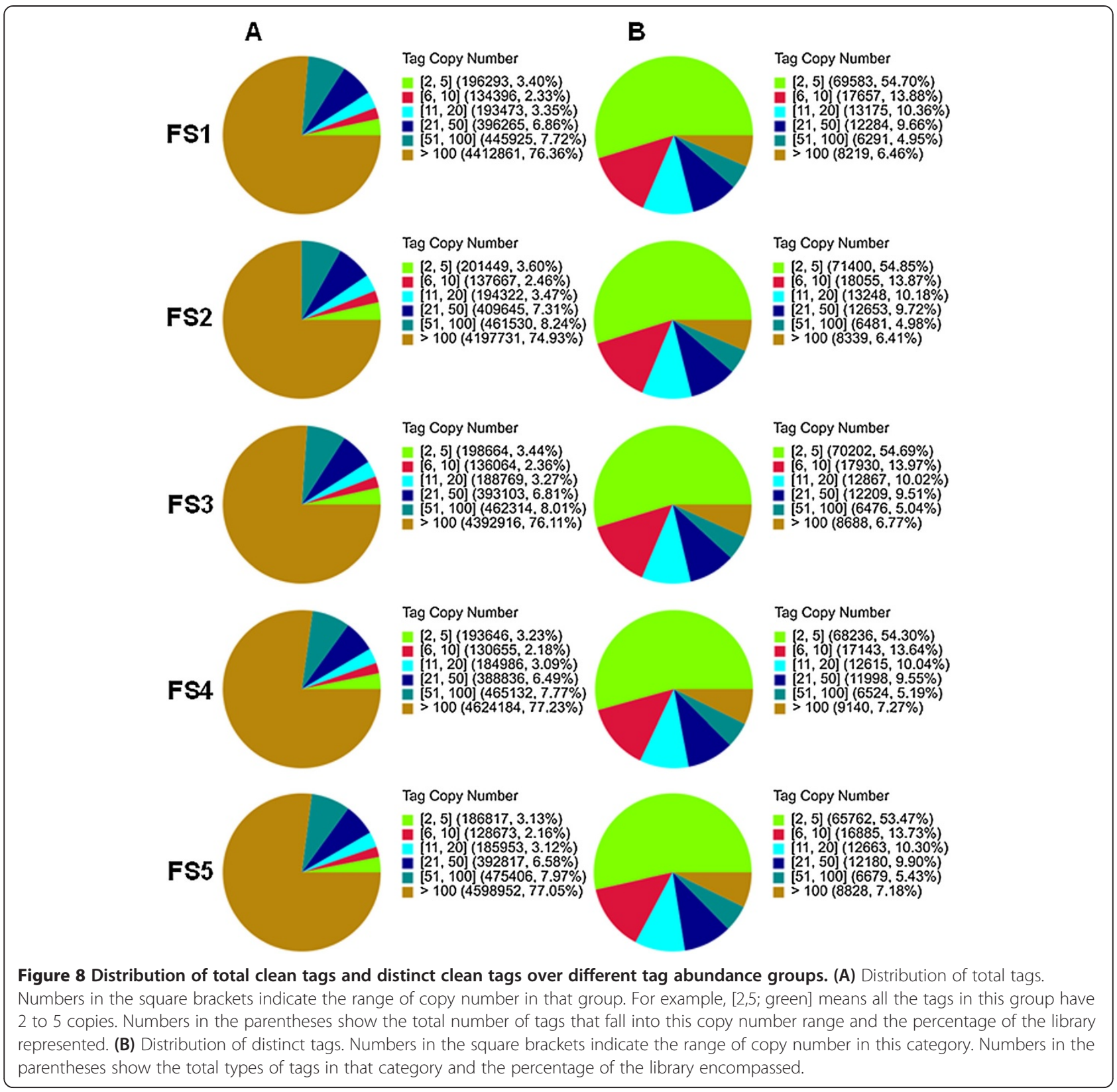

without annotation (Additional file 12). Based on the GO functional classification, almost all genes were involved in cellular components, i.e., extracellular region, membrane part, anchored to membrane and external encapsulating structure. Among genes enriched in $\mathrm{KO}$, significant changes were observed in genes of metabolic pathways, such as biosynthesis of phenylpropanoids, biosynthesis of plant hormones, flavonoid biosynthesis, and starch and sucrose metabolism (Additional file 14).

Between the FS4 and FS3 libraries, 1,824 genes demonstrated significant changes. In the FS4 library, 797 and 1,027 genes were up-regulated and down-regulated, respectively, in comparison with the FS3 library (Figure 10, Additional file 4). Nine genes among the ten most differentially up- and down-regulated genes showed no similarity (Additional file 12). Among the up-regulated genes, five were hypothetical protein from Vitis vinifera and Populus trichocarpa, in which only one matched a flavonoid 3-hydroxylase gene from Ricinus communis, two down-regulated genes were predicted to encode proteins found in Ricinus communis and Populus trichocarpa, an unnamed protein product [Vitis vinifera], a laccase 1a [Populus trichocarpa] and an Nonspecific lipid-transfer protein precursor [Ricinus communis] (Additional file 12). 

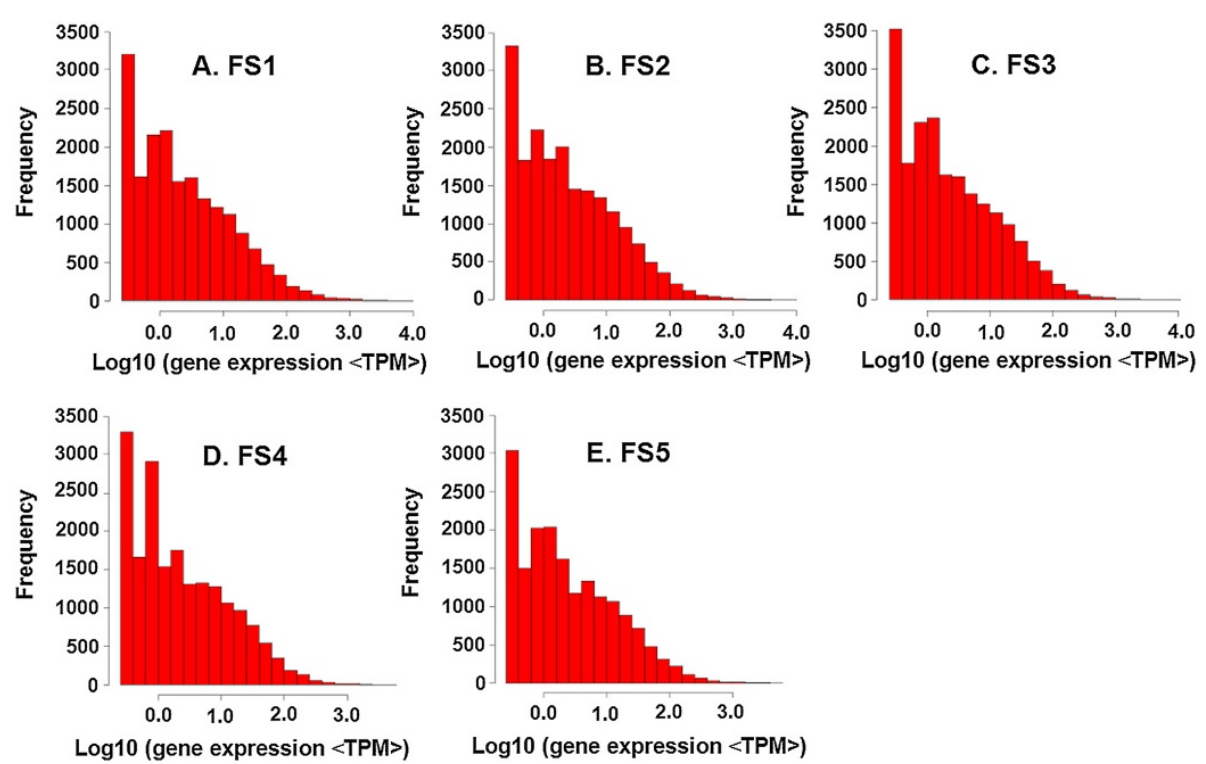

Figure 9 The level of expression for each gene. Gene expression level was determined by calculating the number of unambiguous tags for each gene and then normalized to TPM (transcript copies per million tags). FS1: fruit stage 1, FS2: fruit stage 2, FS3: fruit stage 3, FS4: fruit stage 4, and FS5: fruit stage 5 .

Almost all gene sets enriched in GO were correlated to plastid, plastid part, thylakoid, and chloroplast, while the gene sets enriched in $\mathrm{KO}$ were related to metabolic pathways (Additional file 15).

The comparison between the FS5 and FS4 libraries also revealed significant variations in gene expression. A total of 1,411 genes, including 484 up-regulated and 927 down-regulated, were identified in FS5 compared to FS4 (Figure 10, Additional file 5). Eight genes among the ten most differentially up- and down-regulated genes showed no similarity (Additional file 12). Among the ten

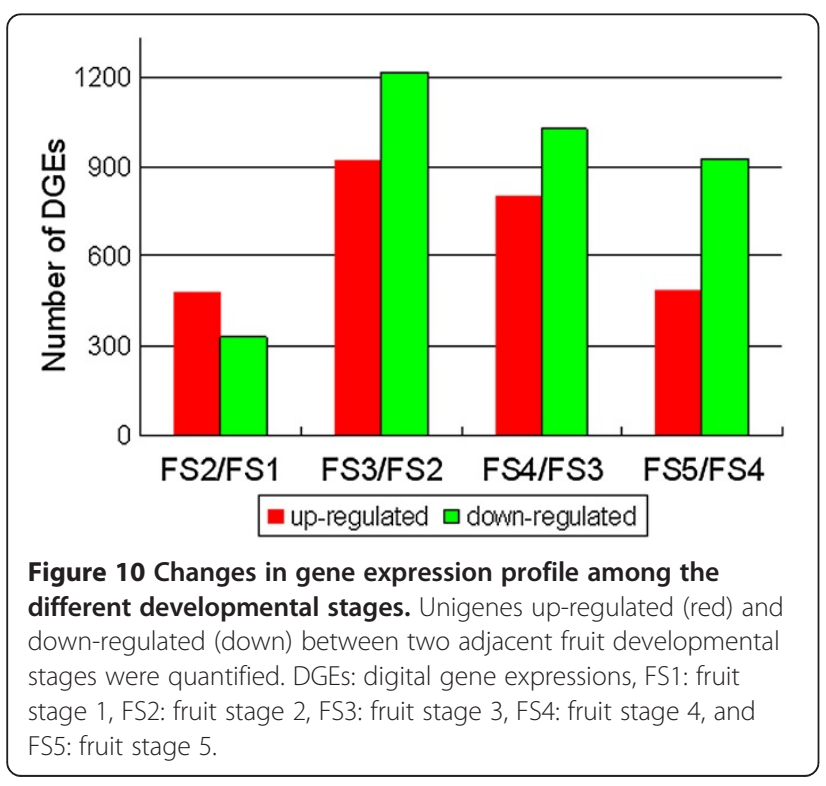

up-regulated genes, one aligned with a beta-D-xylosidase gene [Pyrus pyrifolia], one with an AP2 domain class transcription factor [Malus $\mathrm{x}$ domestica], and two genes were unnamed protein product [Vitis vinifera] and conserved hypothetical protein [Ricinus communis]. Among the ten down-regulated genes, three aligned with dehydration-responsive protein RD22, two with prunin precursors, one with a delta-12 oleate desaturase [Gossypium hirsutum], and two were unnamed protein product or hypothetical protein. Most genes enriched in GO were related (or functional in) chloroplast stroma and photosystem. Genes in enriched KO showing significant changes between the FS5 and FS4 libraries were related to cysteine and methionine metabolism, photosynthesisantenna proteins and isoquinoline alkaloid biosynthesis (Additional file 16).

\section{Expression profiling during ripening}

In this study, differences in the number and expression profile of DEGs were observed at five developmental stages of pear fruit. 28,743 unigenes were expressed during fruit ripening, with $810,2,135,1,824$, and 1,411 showing differential expression between 25 and 55 DPA, 55 and 85 DPA, 85 and 115 DPA, and 115 and 145 DPA, respectively. It showed that fewer genes expressed in the cell division stage (25 to $55 \mathrm{DPA}$ ) than in the period of cell expansion (55 to $145 \mathrm{DPA}$ ) and fruit ripening (115 to $145 \mathrm{DPA}$ ) (Figures 1 and 10).

To characterize the levels of gene expression, the number of unambiguous tags of each gene was calculated and normalized to the number of TPM. Based on 
this analysis, the gene expression levels in the five fruit developmental stages were categorized to rare (TPM < 5), low (TPM > 5-50), moderate (TPM > 50-100), and high (TPM >100) (Additional file 17). The largest portion of transcripts $(20,431$ out of $28,744,71.08 \%)$ exhibited rare expression, and followed by low expression $(6,483$ out of $28,744,22.55 \%)$. Only a small fraction of transcripts was expressed at moderate $(3.21 \%)$ and high (3.15\%) levels.

For a global view of expression patterns, 4 groups were defined according to their expression profiles and some of representative genes were selected and shown in Table 5. Genes in Group I are up-regulated during the fruit development period, which suggests that a few metabolic processes are enhanced and catalytic activity increases. Some genes involved in the process of fruit maturation have this kind of expression patterns, such as SPS, starch synthase, alkaline invertase (Alnv), phosphofructokinase, maltose transporter that are related to starch and sucrose metabolism, 4-hydroxyphenylpyruvate dioxygenase, leucoanthocyanidin dioxygenase (anthocyanin and lignin metabolism), expansin (cell wall loosening protein), and ACC oxidase (ethylene biosynthesis). Genes in Group II are down-regulated, such as genes involved in auxin transport (auxin influx transport protein), sugar metabolism (SS, pyrophosphate-dependent phosphofructo1-kinase), volatile biosynthesis (alcohol acyl-transferase), and phenylpropanoid pathway (arogenate/prephenate dehydratase, UDPG flavonoid 7-O-glucosyltransferase). Genes in Group III showed a high level of gene expression in the middle period of fruit development. These genes are involved in sugar metabolism (NAD-dependent sorbitol dehydrogenase), phytohormone metabolism (DELLA protein, gibberellin oxidase), stress resistance (salt-tolerance protein, polyphenol oxidase), and flavonoid metabolism (p-hydroxyphenylpyruvate dioxygenase, cytochrome P450). Genes in Group IV constitutively expressed through the entire fruit developmental period, such as genes that participate in basal metabolism and membrane stability showed this expression pattern. The information on the gene expression level and pattern during the entire fruit development period will help further elucidate gene functions and well understand the molecular mechanisms of fruit development and maturation in pear species.

\section{Discussion}

\section{Illumina sequencing and function annotation}

In this study, one 'Dangshansuli' pear cDNA library and five DGE libraries (from fruit samples collected at 25, 55, 85, 115 and 145 DPA) were constructed using RNA-Seq technology. These constructed libraries were subjected to comparative gene expression studies. As a result, we obtained 90,227 unigenes including 17,619 clusters and 72,608 singletons. 62,077 out of 90,227 assembled unigenes were annotated. Of which, 61,624 were annotated to the nonredundant (nr) NCBI database and 28,114, 33,205, and 24,196 were annotated to GO, COG and KEGG databases, respectively. For the remaining 28,150 unigenes $(31.2 \%$ of all 90,227 assembled unigenes), the absence of significant homology to existing genes could be caused by several factors. Obviously, the length and completeness of the assembled unigenes were one main factor, which can be seen from the increasing proportion of sequences with matches in the NR database as the length of uingenes increased (Figure 3A). However, the assembled unigenes shorter than 500 bp added up to 64,812 (71.8\% of all 90,227 assembled unigenes) (Figure 2B), and some of them were too short to allow statistically meaningful matches. In addition, for some unigenes, lack of homologous sequences in the public databases may indicate specific roles for them in P. bretschneideri.

Unigenes identified through conserved domain annotation showed that most abundant InterPro domains/families were Protein kinase and its subcategories that are known to regulate the majority of cellular pathways. Cytochrome P450, Myb-DNA-binding families (MYB), and "WD40repeat" domains were also very abundant. MYB and WD40-repeat proteins contributed to the extensive modification and regulation of various secondary compounds in many pathways have been validated to be key roles in anthocyanin biosynthesis by regulating anthocyanin structural genes [48]. MYB proteins also appear to be versatile in regulating secondary metabolism, cellular morphogenesis, meristem formation, and the cell cycle [49]. Recent research showed that Cytochrome P450 encoded enzymes that are responsible for the conversion of the mogroside backbone to various mogrosides [26].

ESTs sequences had been intensively used for transcriptional analysis, candidate gene discovery, and gene functional analysis. However, since RNA-Seq technology was developed, only 4,413 expressed sequence tags (ESTs) of Pyrus plants have been deposited in GenBank due to the fact that EST analyses can only identify limited number of candidate genes that are involved in complex biosynthetic pathways. Using RNA-seq, over one billion nucleotides of high-quality DNA sequence per analysis/experiment can be generated [8], which has dramatically improved the efficiency of gene discovery and functional analysis $[9,10]$. Furthermore, RNA-Seq was less expensive, more efficient, and allowed faster gene discovery than traditional EST analysis. RNA-seq recently has been widely used for transcriptome profiling analysis in many plant species [15,26,41,48-52].

\section{Genes related to metabolic pathway}

In the present study, a total of 31,215 unigenes were assigned to 121 KEGG pathways, among which 2,417 
Table 5 Selected genes with different expression trends in five fruit developmental stages

\begin{tabular}{|c|c|c|c|c|c|c|}
\hline \multirow{2}{*}{ Gene ID } & \multirow{2}{*}{ Gene annotation } & \multicolumn{5}{|c|}{ TPM (transcript per million clean tag) } \\
\hline & & FS1 & FS2 & FS3 & FS4 & FS5 \\
\hline & Up-regulated pattern & & & & & \\
\hline Unigene86203_PB_AHAU & Sucrose phosphate synthase & 0.35 & 0.54 & 1.39 & 1.84 & 0.84 \\
\hline Unigene51453_PB_AHAU & Starch synthase & 6.75 & 11.07 & 21.31 & 9.69 & 30.83 \\
\hline Unigene28216_PB_AHAU & Alkaline invertase & 66.27 & 107.1 & 149.35 & 209.27 & 190.33 \\
\hline Unigene31510_PB_AHAU & Phosphofructokinase & 1.21 & 0.89 & 15.25 & 21.55 & 25.8 \\
\hline Unigene79028_PB_AHAU & Maltose transporter & 30.11 & 34.45 & 35.17 & 72.65 & 123.31 \\
\hline Unigene7706_PB_AHAU & Aldehyde dehydrogenase & 48.28 & 59.44 & 87.67 & 93.03 & 102.54 \\
\hline Unigene11393_PB_AHAU & Expansin & 21.63 & 20.35 & 39.33 & 74.99 & 148.11 \\
\hline Unigene84426_PB_AHAU & ACC oxidase & 8.82 & 13.57 & 13.69 & 6.68 & 159 \\
\hline Unigene41650_PB_AHAU & 4-Hydroxyphenylpyruvate dioxygenase & 0 & 0.54 & 3.47 & 4.01 & 5.36 \\
\hline \multirow[t]{2}{*}{ Unigene49425_PB_AHAU } & Leucoanthocyanidin dioxygenase & 1.73 & 11.07 & 13.34 & 49.77 & 36.19 \\
\hline & Down-regulated pattern & & & & & \\
\hline Unigene78545_PB_AHAU & Auxin influx transport protein & 130.12 & 120.31 & 136.7 & 65.97 & 32.84 \\
\hline Unigene28595_PB_AHAU & Sucrose synthase & 1120.57 & 775.21 & 201.84 & 18.2 & 5.7 \\
\hline Unigene2239_PB_AHAU & Pyrophosphate-dependent phosphofructo-1-kinase & 28.38 & 16.42 & 2.08 & 3.01 & 1.01 \\
\hline Unigene82187_PB_AHAU & Alcohol acyl-transferase & 179.78 & 215.27 & 80.22 & 12.86 & 19.43 \\
\hline Unigene21328_PB_AHAU & Alcohol acyl-transferase & 185.49 & 154.04 & 102.91 & 14.53 & 9.55 \\
\hline Unigene41185_PB_AHAU & Alcohol acyl-transferase & 958.95 & 738.98 & 286.74 & 201.42 & 84.78 \\
\hline Unigene24664_PB_AHAU & Flavonoid 3-hydroxylase & 114.55 & 62.47 & 0 & 3.67 & 0.34 \\
\hline Unigene89425_PB_AHAU & COL domain class transcription factor & 100.88 & 80.86 & 13.34 & 10.52 & 4.36 \\
\hline Unigene40707_PB_AHAU & Arogenate/prephenate dehydratase & 309.04 & 207.23 & 45.74 & 17.04 & 9.21 \\
\hline \multirow[t]{2}{*}{ Unigene42300_PB_AHAU } & UDPG flavonoid 7-O-glucosyltransferase & 125.62 & 192.42 & 72.25 & 34.07 & 67.52 \\
\hline & Low-high-low pattern & & & & & \\
\hline Unigene28017_PB_AHAU & NAD-dependent sorbitol dehydrogenase & 9.52 & 6.96 & 21.66 & 11.69 & 4.02 \\
\hline Unigene48458_PB_AHAU & NAD-dependent sorbitol dehydrogenase & 65.58 & 60.15 & 208.43 & 94.87 & 28.15 \\
\hline Unigene40996_PB_AHAU & p-Hydroxyphenylpyruvate dioxygenase & 0.52 & 1.25 & 12.13 & 6.68 & 8.88 \\
\hline Unigene36705_PB_AHAU & Cytochrome P450 & 36.68 & 39.98 & 208.77 & 167.35 & 69.7 \\
\hline Unigene1335_PB_AHAU & Cytochrome P450 & 12.46 & 8.21 & 77.96 & 21.38 & 11.9 \\
\hline Unigene15706_PB_AHAU & Salt-tolerance protein & 14.36 & 16.78 & 83.86 & 18.2 & 13.57 \\
\hline Unigene6651_PB_AHAU & DELLA protein & 44.99 & 40.7 & 80.74 & 32.4 & 51.44 \\
\hline Unigene29424_PB_AHAU & Polyphenol oxidase & 33.57 & 28.02 & 79.52 & 40.92 & 14.07 \\
\hline \multirow[t]{2}{*}{ Unigene2416_PB_AHAU } & Gibberellin oxidase & 0.35 & 1.61 & 77.96 & 17.7 & 0 \\
\hline & Constitutive pattern & & & & & \\
\hline Unigene1535_PB_AHAU & S6 ribosomal protein & 650.26 & 506.57 & 717.1 & 432.91 & 403.28 \\
\hline Unigene42402_PB_AHAU & Structural molecule activity & 505.09 & 381.09 & 387.23 & 437.58 & 380.99 \\
\hline Unigene41042_PB_AHAU & DEAD-box RNA helicase-like protein & 481.38 & 399.83 & 458.09 & 641.68 & 533.46 \\
\hline Unigene41056_PB_AHAU & Large ribosomal subunit & 333.09 & 256.14 & 291.59 & 243.18 & 225.51 \\
\hline Unigene40598_PB_AHAU & Plasma membrane ATPase & 29.93 & 22.13 & 20.62 & 50.1 & 13.57 \\
\hline Unigene41711_PB_AHAU & Plasma membrane intrinsic protein & 19.38 & 28.92 & 41.23 & 23.88 & 21.11 \\
\hline Unigene8634_PB_AHAU & Plasma intrinsic protein & 1121.95 & 866.6 & 964.51 & 974.21 & 799.18 \\
\hline Unigene13819_PB_AHAU & BHLH domain class transcription factor & 212.49 & 243.11 & 169.79 & 168.85 & 206.92 \\
\hline
\end{tabular}


Table 5 Selected genes with different expression trends in five fruit developmental stages (Continued)

\begin{tabular}{|c|c|c|c|c|c|c|}
\hline Unigene3327_PB_AHAU & $60 S$ ribosomal protein $\mathrm{L} 35 \mathrm{a}$ & 180.65 & 182.6 & 166.5 & 154.99 & 138.73 \\
\hline Unigene40848_PB_AHAU & Ribosomal protein S21e & 173.03 & 218.12 & 188.33 & 170.36 & 182.62 \\
\hline
\end{tabular}

Notes: Gene ID is the accession number of each assembled unigenes deposited in the Transcriptome Shotgun Assembly Sequence Database (TSA) at NCBI.

Abbreviations: DGE, digital gene expression; FS1, fruit stage 1; FS2, fruit stage 2; FS3, fruit stage 3; FS4, fruit stage 4; FS5, fruit stage 5.

unigenes were identified to be associated with carbohydrate metabolisms. A total of $34.8 \%$ and $29.2 \%$ of all 2,417 unigenes are involved in flavonoid biosynthesis and starch and sucrose metabolism, respectively. Invertase (25 unigenes), SS (24 unigenes), ADP glucose pyrophosphorylase (24 unigenes), SPS (23 unigenes), and starch synthase (23 unigenes) were ranked top five enzymes in 18 key enzymes (see Additional file 10) involved in starch and sucrose metabolism pathways.

Sugar is one of the most important biochemical components that determine fruit quality. A series of enzymes control sucrose metabolism during fruit development and maturation. Invertase, SS, and SPS are three key enzymes that are deeply involved in fruit sucrose matabolism. For example, invertase ( $\beta$-D-fructofuranosidase) cleaves sucrose to glucose and fructose irreversible, while soluble acid invertase (AIV) presumably hydrolyzes sucrose to hexose for cell growth and development [53,54]. A decrease of soluble AIV activity was correlated with a rapid increase of sucrose during fruit maturation in Japanese pear $[55,56]$. SS is involved in the movement of sucrose to diverse pathways important for metabolic structure and storage functions in plant cells [57]. It has been reported that SS plays a role in sucrose cleavage rather than sucrose synthesis [58,59]; however, one report suggested that SS was also involved in sucrose synthesis in mature peach fruit where a high level of sucrose was accumulated [60]. In pear fruit, SS also appears to be involved in sucrose synthesis since SS activity increased along with a sucrose accumulation in pear fruit [60]. Recent research in potato also showed that SS strongly determines the intracellular levels of UDP-glucose, ADP-glucose, and starch, and total yield in potato tubers [61]. SPS also plays a major role in sucrose biosynthesis. Both SPS and SS are two important determinants of sucrose accumulation in Asian pear fruit. In 23 pear cultivars, the activity of SS was closely correlated with sucrose content, while SPS showed a weak correlation [60].

In the family Rosaceae, sorbitol is a major carbohydrate of translocated photosynthates. Sorbitol is converted into other sugars in the fruit by sorbitol-metabolizing enzymes, in which sorbitol oxidase and NAD-dependent sorbitol dehydrogenase are two major players $[62,63]$. Research also revealed that three gene families, sorbitol transport (SOT), sorbitol dehydrogenase (SDH), and sorbitol-6-phosphate dehydrogenase (S6PDH), showed more impacts on sugar metabolism in pear than in non-rosaceous species [64]. Wu et al. (2013) [64] identified four S6PDH genes through genome sequencing; however, no unigenes coding $\mathrm{S} 6 \mathrm{PDH}$ were detected in this study. This may be caused by the difference in gene assembly or annotation. Yamaki and Moriguchi (1989) reported that NAD-dependent sorbitol dehydrogenase that converts sorbitol to fructose showed a high activity throughout the entire fruit developing period in Japanese pear fruit. However, the activity of sorbitol oxidase activity that is about one-tenth of NADdependent sorbitol dehydrogenase, was high in immature fruit, but decreased during the fruit expansion period and increased again during the fruit maturation stage [63]. In peach fruit, sorbitol oxidase activity was relatively high, but other sorbitol-related enzymes were barely detectable [65].

In this study, two important enzymes, ADP glucose pyrophosphorylase (24 unigenes) and starch synthase (23 unigenes) that are involved in starch metabolic pathways were identified. In plants, regulation of starch metabolism is complex. In synthesis of starch, ADP-glucose was the glucosyl donor for the elongation of $\alpha-1,4$-glucosidic chains [66]. The first committed step is ADP-glucose synthesis that is catalyzed by ADP-glucose pyrophosphorylase (ADPGlc PPase) [67]. As a glucose donor, ADPG molecule is transferred to non-reducing end of the (1-4) glucan primer by starch synthase catalyses, thus formation of a long-chain amylase. Ghosh and Preiss (1966) showed that the reaction catalyzed by ADPGlc PPase is a regulation step in starch synthesis in higher plants as well as in the cyanobacteria [68-70]. Most of the plant ADPGlc PPases are allosterically regulated by 3-PGA and inorganic orthophosphate (Pi) $[69,70]$. Some reports also suggested that in higher plants the enzyme activity can also be regulated by its reductive state [71-73].

In this study, multiple unigenes in the main flavonoid biosynthesis pathways were annotated according to KEGG (Figure 6B). Flavonoids including phenylpropanoids, flavones, flavonols, and anthocyanins are important secondary metabolites that are directly involved in the development of fruit quality, such as color, flavor, and health beneficial ingredients $[1,46,74]$. These compounds are also involved in the formation of undesirable brown pigments in fruits following bruising or cutting and/or storage [75]. Information on phenylpropanoid metabolism in pear is limited. Nishitani et al. (2010) have studied the importance of phenylpropanoid metabolism in pear fruit ripening using oligoarray analysis [75]. In present study, a large number (497) of unigenes involved in phenylpropanoid biosynthesis were detected. Lignin biosynthesis in the phenylpropanoid pathway 
is one of the important factors for pear fruit quality. Lignin is the primary component of stone cells in pear fruit [76] and its synthesis has direct influence on formation and content of stone cells, ultimately influencing pear fruit quality [64]. Anthocyanin biosynthesis is essential for fruit coloration. Red coloration is determined by the content and composition of anthocyanins in the fruit skin [77]. Most enzymes in the anthocyanin biosynthetic pathway in pears are well studied [76]. For example, Feng et al. (2010) reported that anthocyanin biosynthesis in pears is regulated by a R2R3-MYB transcription factor PyMYB10 [78].

Digital gene expression (DGE) at different stages of pear fruits Analyses of KEGG pathways showed that DEGs were observed in several different pathways including some metabolic pathways, such as biosynthesis of phenylpropanoids, plant hormones, and flavonoids; starch and sucrose metabolism; cysteine and methionine metabolism; biosynthesis of photosynthesis-antenna proteins and isoquinoline alkaloid. These are closely related to fruit development in pear and other species $[60,61,66,74,79]$.

Comparative analysis showed that dehydration-responsive protein RD22 was significantly up-regulated in pear fruit between FS3 and FS2 and significantly down-regulated between the FS3 and FS2 libraries. This showed that this gene involved in the whole expansion stage of pear fruit, so we infer that it may play important roles in the process of pear fruit growth and development. Although some research reported that dehydration-responsive protein RD22 was related to stress/defense response [80-82]. The role of this gene in pear fruit development remains unknown. AP2 was well known for its roles in floral organ identity and develop. In this study, we found that AP2 domain class transcription factor was significantly down-regulated and up-regulated in the fruit developmental stage of FS3/FS2 and FS5/FS4, respectively, which indicated that the AP2 domain class transcription factor was involved in fruit growth and development in pear. Recent research showed that AP2 was involved in many aspects of fruit development in other species. For example, Chung et al. (2010) proved that a tomato (Solanum lycopersicum) APETALA2/ERF gene (SIAP2a) is a negative regulator of fruit ripening [83]. AP2 genes are also involved in seed mass and yield development via regulation of embryo cell number and cell size [84]. Rohrmann et al. (2011) reported that some genes in the AP2-EREBP family responsive to ethylene also showed the altered expression from the green fruit developmental stage onwards [85]. Ripoll et al. (2011) found that AP2 acts to prevent overgrowth of replum by negatively regulating $\mathrm{BP}$ and $\mathrm{RPL}$, two genes that normally act to promote replum formation in Arabidopsis. AP2 also acts to prevent overgrowth of the valve margin by repressing the expression of the valve margin identity gene [86]. These studies indicated that AP2 domain class transcription factor is an important regulatory factor in fruit development. Other genes identified in this study, such as metallothionein-like protein type 3, nonspecific lipid-transfer protein precursor, cytochrome $\mathrm{P} 450$, zinc finger protein, prunin 2 precursor, flavonoid 3hydroxylase gene, CBF/DREB1 transcription factor, anthocyanidin synthase gene, etc. may have their own roles in the process of pear fruit growth and development. These roles need further verification.

\section{Conclusions}

In this study, the transcriptome profile of Chinese white pear ( $P$. bretschneideri) was investigated using Solexa/Illumina RNA-seq and DGE deep sequencing technologies. A total of 90,227 unigenes were assembled and 62,077 unigenes were annotated. The results demonstrated the feasibility of using Illumina sequencing-based DGE system for gene expression profiling and provided new directions for functional analysis of genes involved in pear fruit development. These findings provide a substantial contribution to existing sequence resources of pear species and will certainly valuable for elucidation of molecular mechanisms in fruit development and maturation in pear or related species. Therefore, this study clearly evidenced that Illumina sequencing technology could be applied as a rapid and costeffective method for de novo transcriptome analysis of nonmodel plant species that lack of prior genome annotation.

\section{Availability of supporting data}

The assembled unigenes (larger than 200 bp) were deposited in the Transcriptome Shotgun Assembly Sequence Database (TSA) at NCBI, with accession numbers from JR595427 to JR673747.

\section{Additional files}

Additional file 1: Top BLAST hits from NCBI nr database. BLAST results against the NCBI nr database for all distinct sequences with a cut-off $E$ value above $10^{-5}$

Additional file 2: Differentially expressed genes between fruit stage 2 (FS2) and fruit stage 1 (FS1). TPM: transcript copies per million tags. Raw intensity: the total number of tags sequenced for each gene. FDR: false discovery rate. FDR $<0.001$ and the absolute value of log2Ratio $\leq 1$ were used as the threshold to judge the significance of gene expression difference. In order to calculate the log2Ratio and FDR, TPM value of 0.001 instead of 0 for genes that do not express in one sample was used.

Additional file 3: Differentially expressed genes between fruit stage 3 (FS3) and fruit stage 2 (FS2). TPM: transcript copies per million tags. Raw intensity: the total number of tags sequenced for each gene. FDR: false discovery rate. FDR $<0.001$ and the absolute value of log2Ratio $\leq 1$ were used as the threshold to judge the significance of gene expression difference. In order to calculate the log2Ratio and FDR, TPM value of 0.001 instead of 0 for genes that do not express in one sample was used.

Additional file 4: Differentially expressed genes between fruit stage 4 (FS4) and fruit stage 3 (FS3). TPM: transcript copies per million tags. Raw intensity: the total number of tags sequenced for each gene. FDR: false discovery rate. FDR $<0.001$ and the absolute value of log2Ratio $\leq 1$ were used as the threshold to judge the significance of gene expression difference. In order to calculate the 
$\log 2$ Ratio and FDR, TPM value of 0.001 instead of 0 for genes that do not express in one sample was used.

Additional file 5: Differentially expressed genes between fruit stage 5 (FS5) and fruit stage 4 (FS4). TPM: transcript copies per million tags. Raw intensity: the total number of tags sequenced for each gene. FDR: false discovery rate. FDR $<0.001$ and the absolute value of log2Ratio $\leq 1$ were used as the threshold to judge the significance of gene expression difference. In order to calculate the log2Ratio and FDR, TPM value of 0.001 instead of 0 for genes that do not express in one sample was used.

Additional file 6: Random distribution of Illumina sequencing reads in the assembled unigenes. The $x$-axis indicates the relative position of sequencing reads in the assembled unigenes. The orientation of unigene is from $5^{\prime}$ to $3^{\prime}$ end.

Additional file 7: $\mathrm{GO}$ annotation of unigenes. Combination of the $\mathrm{GO}$ annotation provided by Blast2Go and InterProScan

Additional file 8: COG Functional classification of the $P$. bretschneideri transcriptome. A total of 33,205 unigenes showed significant similarity to one of the 25 COG categories at NCBI (E-value $\left.\leq 1.0 \mathrm{e}^{-5}\right)$.

Additional file 9: Kyoto Encyclopedia of Genes and Genomes (KEGG) ontology $(\mathrm{KO})$ classification of the $P$. bretschneideri transcriptome. A total of 24,169 sequences were assigned to 121 KEGG pathways.

Additional file 10: Putative genes coding for structural enzymes in key pathways of primary, intermediate, and secondary metabolism in the P. bretschneideri datasets.

Additional file 11: Correlation between the number of detected genes and sequencing volume (total tag number). All figures show a trend of saturation. Once sequencing reaches 4 million reads, the number of detected genes almost ceases to increase.

Additional file 12: Top ten differentially expressed genes in each twoway library comparison.

Additional file 13: Gene set enrichment analysis comparing FS2 and FS1. Additional file 14: Gene set enrichment analysis comparing FS3 and FS2. Additional file 15: Gene set enrichment analysis comparing FS4 and FS3. Additional file 16: Gene set enrichment analysis comparing FS5 and FS4. Additional file 17: Differentially expressed genes of five fruit developmental stages. TPM: transcript copies per million tags.

\section{Competing interests}

The authors declare that they have no competing interests.

\section{Authors' contributions}

CBF, WHD and OY conceived and designed the experiment. YH and XW participated in sample collection and RNA preparation. MX, YH, HY and CBF analyzed and interpreted the sequence data. MX, YPZ, YH and HY deposited the sequencing data in the GenBank database. CBF, YPZ, OY and WHD drafted and revised the manuscript. All authors read and approved the final manuscript.

\section{Acknowledgements}

The project was funded by the National Natural Science Foundation of China (grants no. 31071756 and 31201583), and the Program for Innovative Research Team in Anhui Agricultural University.

\section{Author details}

${ }^{1}$ School of Horticulture, Anhui Agricultural University, Hefei 230036, Anhui, P R China. ${ }^{2}$ Key Laboratory of Tea Biochemistry \& Biotechnology, Ministry of Education, Anhui Agricultural University, Hefei 230036, Anhui, P R China. ${ }^{3}$ Department of Plant Sciences, North Dakota State University, Fargo, ND 58108, USA.

Received: 9 August 2013 Accepted: 20 November 2013

Published: 23 November 2013

\section{References}

1. Bell RL, Quamme HA, Layne REC, Skirvin RM: Pears. In Fruit breeding, volume I: tree and tropical fruits. Edited by Janick J, Moore JN. UK: John Wiley and Sons Inc; 1996:441-514.

2. Bell RL: Pears (Pyrus). In Genetic resources of temperate fruit and nut crops I. Edited by Moore JN, Ballington JR Jr. Wageningen, The Netherlands: International Society for Horticultural Science; 1990:655-697.

3. Zhang XD, Andrew CA, Yi Q, Chen LM, Li KZ, Shu Q, Su J: Differential gene expression analysis of Yunnan red pear, Pyrus pyrifolia, during fruit skin coloration. Plant Mol Biol Rep 2011, 29:305-314.

4. Nishitani C, Terakami S, Sawamura Y, Takada N, Yamamoto T: Development of novel EST-SSR markers derived from Japanese pear (Pyrus pyrifolia). Breed Sci 2009, 59:391-400.

5. Zhang HL, Wang YJ, Zhang CH, Wang XP, Li HE, Xu WR: Isolation, characterization and expression analysis of resistance gene candidates in pear (Pyrus spp). Sci Hortic 2011, 127:282-289.

6. Nahla B, Joseph DP: Identification of European and Asian pears using EST-SSRs from Pyrus. Genet Resour Crop Evol 2010, 57:357-370.

7. Javier S: Genomic characterization of self-incompatibility ribonucleases (S-RNases) in European pear cultivars and development of PCR detection for 20 alleles. Tree Genetics \& Genomes 2009, 5:393-405.

8. Huang W, Marth G: EagleView: a genome assembly viewer for next generation sequencing technologies. Genome Res 2008, 18:1538-1543.

9. Schuster SC: Next-generation sequencing transforms today's biology. Nat Methods 2008, 5:16-18.

10. Ansorge WJ: Next-generation DNA sequencing techniques. N Biotechnol 2009, 25:195-203.

11. Wang Z, Gerstein M, Snyder M: RNA-Seq: a revolutionary tool for transcriptomics. Nat Rev Genet 2009, 10:57-63.

12. Morozova O, Marra MA: Applications of next-generation sequencing technologies in functional genomics. Genomics 2008, 92:255-264.

13. Nagalakshmi U, Wang Z, Waern K, Shou C, Raha D, Gerstein M, Snyder M: The transcriptional landscape of the yeast genome defined by RNA sequencing. Science 2008, 320:1344-1349.

14. Wilhelm BT, Marguerat S, Watt S, Schubert F, Wood V, Goodhead I, Penkett CJ, Rogers J, Bähler J: Dynamic repertoire of a eukaryotic transcriptome surveyed at single-nucleotide resolution. Nature 2008, 453:1239-1243.

15. Lister R, O'Malley RC, Tonti-Filippini J, Gregory BD, Berry CC, Millar AH, Ecker JA: Highly integrated single-base resolution maps of the epigenome in Arabidopsis. Cell 2008, 133:523-536.

16. Cloonan N, Forrest ARR, Kolle G, Gardiner BBA, Faulkner GJ, Brown MK, Taylor DF, Steptoe AL, Wani S, Bethel G: Stem cell transcriptome profiling via massive-scale mRNA sequencing. Nat Methods 2008, 5:613-619.

17. Mortazavi A, Williams BA, McCue K, Schaeffer L, Wold B: Mapping and quantifying mammalian transcriptomes by RNA-Seq. Nat Methods 2008, 5:621-628.

18. Marioni J, Mason C, Mane S, Stephens M, Gilad Y: RNA-seq: an assessment of technical reproducibility and comparison with gene expression arrays. Genome Res 2008, 18:1509-1517.

19. Sultan M, Schulz MH, Richard H, Magen A, Klingenhoff A, Scherf M, Seifert M, Borodina T, Soldatov A, Parkhomchuk D, Schmidt D, O'Keeffe S, Haas S, Vingron $M$, Lehrach $H$, Yaspo ML: A global view of gene activity and alternative splicing by deep sequencing of the human transcriptome. Science 2008, 321:956-960.

20. Liu GQ, Li WS, Zheng PH, Xu T, Chen LJ, Liu DF, Hussain S, Teng YW: Transcriptomic analysis of 'Suli' pear (Pyrus pyrifolia white pear group) buds during the dormancy by RNA-Seq. BMC Genomics 2012, 13:700.

21. Morin R, Bainbridge $M$, Fejes A, Hirst M, Krzywinski M, Pugh T, McDonald $H$ Varhol R, Jones S, Marra M: Profiling the HeLa S3 transcriptome using randomly primed cDNA and massively parallel short-read sequencing. Biotechniques 2008, 45:81-94.

22. Vera JC, Wheat CW, Fescemyer HW, Frilander MJ, Crawford DL, Hanski I, Marden JH: Rapid transcriptome characterization for a nonmodel organism using 454 pyrosequencing. Mol Ecol 2008, 17:1636-1647.

23. Kristiansson E, Asker N, Forlin L, Larsson DGJ: Characterization of the Zoarces viviparus liver transcriptome using massively parallel pyrosequencing. BMC Genomics 2009, 10:345.

24. Meyer E, Aglyamova GV, Wang S, Buchanan-Carter J, Abrego D, Colbourne JK, Willis BL, Matz MV: Sequencing and de novo analysis of a coral larval transcriptome using 454 GSFIx. BMC Genomics 2009, 10:219. 
25. Wang XW, Luan JB, Li JM, Bao YY, Zhang CX, Liu SS: De novo characterization of a whitefly transcriptome and analysis its gene expression during development. BMC Genomics 2010, 11:400.

26. Tang Q, Ma XJ, Mo CM, Wilson IW, Song C, Zhao H, Yang YF, Fu W, Qiu DY: An efficient approach to finding Siraitia grosvenorii triterpene biosynthetic genes by RNA-seq and digital gene expression analysis. BMC Genomics 2011, 12:343.

27. Xue J, Bao YY, Li BL, Cheng YB, Peng ZY, Liu H, Xu HJ, Zhu ZR, Lou YG, Cheng JA, Zhang CX: Transcriptome analysis of the brown planthopper Nilaparvata lugens. PloS ONE 2010, 5:e14233.

28. Xiang LX, He D, Dong WR, Zhang YW, Shao JZ: Deep sequencing-based transcriptome profiling analysis of bacteria-challenged Lateolabrax japonicus reveals insight into the immune-relevant genes in marine fish. BMC Genomics 2010, 11:472.

29. Wu J, Zhang $Y L$, Zhang HQ, Huang H, Kevin MF, Lu J: Whole genome wide expression profiles of Vitis amurensis grape responding to downy mildew by using Solexa sequencing technology. BMC Plant Biol 2010, 10:234.

30. Wu T, Qin ZW, Zhou XY, Feng Z, Du YL: Transcriptome profile analysis of floral sex determination in cucumber. J Plant Physiol 2010, 167:905-913.

31. Wang QQ, Liu F, Chen XS, Ma XJ, Zeng HQ, Yang ZM: Transcriptome profiling of early developing cotton fiber by deep-sequencing reveals significantly differential expression of genes in a fuzzless/ lintless mutant. Genomics 2010, 96:369-376.

32. Fátima RC, Noé DF, Jean PVC, Andrés CH, Nayelli MM, Stefan F: A simple and efficient method for isolating small RNAs from different plant species. Plant Methods 2011, 7:4-10.

33. Carra A, Gambino G, Schubert A: A cetyltrimethylammonium bromidebased method to extract low-molecular-weight RNA from polysaccharide-rich plant tissues. Anal Biochem 2007, 360:318-320.

34. Li R, Zhu H, Ruan J, Qian W, Fang X, Shi Z, Li Y, Li S, Shan G, Kristiansen K, Li $\mathrm{S}$, Yang $\mathrm{H}$, Wang J, Wang J: De novo assembly of human genomes with massively parallel short read sequencing. Genome Res 2010, 20:265-272.

35. Pertea G, Huang X, Liang F, Antonescu V, Sultana R, Karamycheva S, Lee Y White J, Cheung F, Parvizi B, Tsai J, Quackenbush J: TIGR gene indices clustering tools (TGICL):a software system for fast clustering of large EST datasets. Bioinformatics 2003, 19:651-652.

36. Conesa A, Gotz S, Garcia-Gomez JM, Terol J, Talon M, Robles M: Blast2GO: a universal tool for annotation, visualization and analysis in functional genomics research. Bioinformatics 2005, 21(18):3674-3676.

37. Ye J, Fang L, Zheng HK, Zhang Y, Chen J, Zhang ZJ, Wang J, Li ST, Li RQ, Bolund L, Wang J: WEGO: a web tool for plotting GO annotations. Nucleic Acids Res 2006, 34:W293-W297.

38. Audic S, Claverie JM: The significance of digital gene expression profiles. Genome Res 1997, 7:986-995.

39. Shi CY, Yang H, Wei CL, Yu O, Zhang ZZ, Jiang CJ, Sun J, Li YY, Chen Q, Xia T, Wan XC: Deep sequencing of the Camellia sinensis transcriptome revealed candidate genes for major metabolic pathways of tea-specific compounds. BMC Genomics 2011, 12:131-139.

40. Wang X, Sun Q, McGrath SD, Mardis ER, Soloway PD, Clark AG: Transcriptome-wide identification of novel imprinted genes in neonatal mouse brain. PLOS ONE 2008, 3:e3839.

41. Feng C, Chen M, Xu CJ, Bai L, Yin XR, Li X, Allan AC, Ferguson IB, Chen KS: Transcriptomic analysis of Chinese bayberry (Myrica rubra) fruit development and ripening using RNA-Seq. BMC Genomics 2012, 13:19.

42. Hunter S, Apweiler R, Attwood TK, Bairoch A, Bateman A, Binns D, Bork P, Das U, Daugherty L, Duquenne L, Finn RD, Gough J, Haft D, Hulo N, Kahn D, Kelly E, Laugraud A, Letunic I, Lonsdale D, Lopez R, Madera M, Maslen J, McAnulla C, McDowall J, Mistry J, Mitchell A, Mulder N, Natale D, Orengo C, Quinn AF, Selengut JD, Sigrist CJ, Thimma M, Thomas PD, Valentin F, Wilson $D, W u C H$, Yeats C: InterPro: the integrative protein signature database. Nucleic Acids Res 2009, 37:D211-D215.

43. Sompompailin K, Makita Y, Saito K: A WD-repeat-containing putative regulatory protein in anthocyanin biosynthesis in Perilla frutescens. Plant Mol Biol 2002, 50:485-495.

44. Finn RD, Mistry J, Tate J, Coggill P, Heger A, Pollington JE, Gavin OL, Gunasekaran P, Ceric G, Forslund K, Holm L, Sonnhammer ELL, Eddy SR, Bateman A: The Pfam protein families database. Nucleic Acids Res 2010, 38:D211-D222.

45. Kanehisa M, Goto S, Kawashima S, Okuno Y, Hattori M: The KEGG resource for deciphering the genome. Nucleic Acids Res 2004, 32:D277-D280.
46. Bell $\mathrm{RL}$, Janick J: Quantitative genetic analysis of fruit quality in pear. J Amer Soc Hort Sci 1990, 115:829-834.

47. Yamamoto T, Chevreau E: Pear genomics. In Plant genetics/ genomics, volume 6: genetics and genomics of rosaceae. Edited by Kevin MF, Susan EG. LLC, USA: Springer Science + Business Media; 2009:163-186

48. Xie FL, Burklew CE, Yang YF, Liu M, Xiao P, Zhang BH, Qiu DY: De novo sequencing and a comprehensive analysis of purple sweet potato (Impomoea batatas L.) transcriptome. Planta 2012, 236:101-113.

49. Jin H, Martin C: Multifunctionality and diversity within the plant MYB-gene family. Plant Mol Biol 1999, 41:577-585.

50. $\mathrm{Xu} \mathrm{H}$, Gao Y, Wang JB: Transcriptomic analysis of rice (Oryza sativa) developing embryos using the RNA-Seq technique. PLOS ONE 2012, 7:e30646.

51. Zhang YG, Zhu J, Dai HY: Characterization of transcriptional differences between columnar and standard apple trees using RNA-Seq. Plant Mol Biol Rep 2012, 30:957-965.

52. Mizrachi E, Hefer CA, Ranik M, Joubert F, Myburg AA: De novo assembled expressed gene catalog of a fast-growing Eucalyptus tree produced by Illumina mRNA-Seq. BMC Genomics 2010, 11:681.

53. Pfeiffer I, Kutschera U: Sucrose metabolism and cell elongation in developing sunflower hypocotyls. J Exp Bot 1995, 46:631-638.

54. Tang X, Ruffner HP, Scholes JD, Rolfe SA: Purification and characterisation of soluble invertases from leaves of Arabidopsis thaliana. Planta 1996, 198:17-23.

55. Moriguchi T, Abe K, Sanada T, Yamaki S: Levels and role of suscrose synthase, sucrose-phosphate synthase, and acid invertase in sucrose accumulation in fruit of Asian pear. J Am Soc Hort Sci 1992, 117:274-278.

56. Tanase K, Yamaki S: Sucrose synthase isozymes related to sucrose accumulation during fruit development of Japanese pear (Pyrus pyrifolia Nakai). J Japan Soc Hort Sci 2000, 69:671-676.

57. Komatsu A, Moriguchi T, Koyama K, Omura M, Akihama T: Analysis of sucrose synthase genes in citrus suggests different roles and phylogenetic relationships. J Exp Bot 2002, 53:61-71.

58. Hawker JS: Sucrose. In Biochemistry of storage carbohydrates in green plants. Edited by Dey PM, Dixon RA. New York, USA: Academic; 1985:1-51.

59. Turner JF, Turner DH: The regulation of carbohydrate metabolism. Annu Rev Plant Physiol 1975, 26:159-186.

60. Moriguchi T, Sanada T, Yamakil S: Seasonal fluctuations of some enzymes relating to sucrose and sorbitol metabolism in peach fruit. J Amer SoC Hort Sci 1990, 115:278-281.

61. Baroja-Fernández E, Munoz FJ, Montero M, Etxeberria E, Sesma MT, Ovecka M, Bahaji A, Ezquer I, Li J, Prat S, Pozueta-Romero J: Enhancing sucrose synthase activity in transgenic potato (Solanum tuberosum L.) tubers results in increased levels of starch, ADPglucose and UDPglucose and total yield. Plant Cell Physiol 2009, 50:1651-1662.

62. Yamaki S, Ishikawa K: Roles of four sorbitol related enzymes and invertase in the seasonal alteration of sugar metabolism in apple tissue. J Amer Soc Hort Sci 1986, 111:134-137.

63. Yamaki S, Moriguchi T: Seasonal fluctuation of sorbitol related enzymes and invertase activities accompanying maturation of Japanese pear (Pyrus serotina Rehder var. cults Rehder) fruit. J Jpn Soc Hort Sci 1989, 57:602-607.

64. Wu J, Wang Z, Shi Z, Zhang S, Ming R, Zhu S, Khan MA, Tao S, Korban SS, Wang $\mathrm{H}$, Chen NJ, Nishio T, et al: The genome of pear (Pyrus bretschneideri Rehd). Genome Res 2012. doi:10.1101/gr.144311.112.

65. Moriguchi T, Sanada T, Yamaki S: Seasonal fluctuations of some enzymes relating to sucrose and sorbitol metabolism in peach fruit. J Amer Hort Sci 1990, 115:278-281.

66. Ballicora MA, Iglesias AA, Preiss J: ADP-glucose pyrophosphorylase: a regulatory enzyme for plant starch synthesis. Photosynth Res 2004 79:1-24

67. Martin C, Smith AM: Starch biosynthesis. Plant Cell 1995, 7:971-985.

68. Ghosh HP, Preiss J: Adenosine diphosphate glucose pyrophosphorylase: a regulatory enzyme in the biosynthesis of starch in spinach leaf chloroplasts. J Biol Chem 1966, 241:4491-4504.

69. Preiss J, Sivak MN: Biochemistry, molecular biology and regulation of starch synthesis. Genet Eng 1998, 20:177-223.

70. Preiss J: Biosynthesis of bacterial and mammalian glycogen and plant starch synthesis and their regulation. In Biorganic chemistry: carbohydrates. Edited by Hecht SM. Oxford: Oxford University Press; 1999:489-554. 
71. Fu Y, Ballicora MA, Leykam JF, Preiss J: Mechanism of reductive activation of potato tuber ADP-glucose pyrophosphorylase. J Biol Chem 1998, 273:25045-25052.

72. Ballicora MA, Frueauf JB, Fu Y, Schurmann P, Preiss J: Activation of the potato tuber ADP-glucose pyrophosphorylase by thioredoxin. J Biol Chem 2000, 275:1315-1320.

73. Tiessen A, Hendriks JH, Stitt M, Branscheid A, Gibon Y, Farre EM, Geigenberger P: Starch synthesis in potato tubers is regulated by posttranslational redox modification of ADP-glucose pyrophosphorylase: a novel regulatory mechanism linking starch synthesis to the sucrose supply. Plant Cell 2002, 14:2191-2213.

74. Fischer TC, Gosch C, Pfeiffer J, Halbwirth H, Halle C, Stich K, Forkmann G: Flavonoid genes of pear (Pyrus communis). Trees 2007, 21:521-529.

75. Nishitani C, Shimizu T, Fujii H, Hosaka F, Terakami S, Nakamura Y, Itai A, Yamaguchi-Nakamura A, Yamamoto T: Oligoarray analysis of gene expression in ripening Japanese pear fruit. Sci Hortic 2010, 124:195-203.

76. Tao S, Khanizadeh S, Zhang H, Zhang S: Anatomy, ultrastructure and lignin distribution of stone cells in two Pyrus species. Plant Sci 2009, 176:413-419.

77. Steyn WJ, Wand SJE, Holcroft DM, Jacobs G: Red colour development and loss in pears. Acta Hortic 2005, 671:79-85.

78. Feng SQ, Wang YL, Yang S, Xu YT, Chen XS: Anthocyanin biosynthesis in pears is regulated by a R2R3-MYB transcription factor PyMYB10. Planta 2010, 232:245-255.

79. Baur AH, Yang SF: Methionine metabolism in apple tissue in relation to ethylene biosynthesis. Phytochemistry 1972, 11:3207-3214.

80. Fernandez L, Torregrosa L, Terrier N, Sreekantan L, Grimplet J, Davies C, Thomas MR, Romieu C, Ageorges A: Identification of genes associated with flesh morphogenesis during grapevine fruit development. Plant $\mathrm{Mol}$ Biol 2007, 63:307-323.

81. Ziosi V, Bonghi C, Bregoli AM, Trainotti L, Biondi S, Sutthiwal S, Kondo S, Costa G, Torrigiani P: Jasmonate-induced transcriptional changes suggest a negative interference with the ripening syndrome in peach fruit. J Exp Bot 2008, 59:563-573.

82. Dhanaraj AL, Alkharouf NW, Beard HS, Chouikha IB, Matthews BF, Wei H, Arora R, Rowland $\mathrm{L}$ : Major diVerences observed in transcript proWles of blueberry during cold acclimation under weld and cold room conditions. Planta 2007, 225:735-751.

83. Chung MY, Vrebalov J, Alba R, Lee J, McQuinn R, Chung JD, Klein P, Giovannoni J: A tomato (Solanum lycopersicum) APETALA2/ERF gene, SIAP2a, is a negative regulator of fruit ripening. Plant J 2010, 64:936-947.

84. Ohto M, Fischer RL, Goldberg RB, Nakamura K, Harada JJ: Control of seed mass by APETALA2. Proc Natl Acad Sci 2005, 102:3123-3128.

85. Rohrmann J, Tohge T, Alba R, Osorio S, Caldana C, McQuinn R, Arvidsson S, Merwe MJ, Rianō-Pachón DM, Mueller-Roeber B, Fei Z, Nesi AN, Giovannoni $\mathrm{J}$, Fernie AR: Combined transcription factor profiling, microarray analysis and metabolite profiling reveals the transcriptional control of metabolic shifts occurring during tomato fruit development. Plant J 2011, 68:999-1013.

86. Ripoll JJ, Roeder AHK, Ditta GS, Yanofsky MF: A novel role for the floral homeotic gene APETALA2 during Arabidopsis fruit development. Development 2011, 138:5167-5176.

doi:10.1186/1471-2164-14-823

Cite this article as: Xie et al.: Transcriptome profiling of fruit development and maturation in Chinese white pear (Pyrus bretschneideri Rehd). BMC Genomics 2013 14:823.

\section{Submit your next manuscript to BioMed Central and take full advantage of:}

- Convenient online submission

- Thorough peer review

- No space constraints or color figure charges

- Immediate publication on acceptance

- Inclusion in PubMed, CAS, Scopus and Google Scholar

- Research which is freely available for redistribution

Submit your manuscript at www.biomedcentral.com/submit
C Biomed Central 